\title{
Short-term effects of gaseous pollutants on cause- specific cerebrovascular disease among patients with type 2 diabetes: case-crossover evidence from Beijing, China
}

\section{Xiangtong Liu}

Capital Medical University

Zhiwei Li

Capital Medical University

Moning Guo

Beijing Public Health Information Center

Feng Lu

Beijing Public Health Information Center

Aklilu Deginet

Capital Medical University

Mengmeng Liu

Capital Medical University

Xiaolin Xu

University of Queensland

Yanxia Luo

Capital Medical University

Yaoyu Hu

Capital Medical University

Yutong Li

Capital Medical University

Mengqiu Wu

Capital Medical University

Mengyang Liu

Capital Medical University

Lixin Tao

Capital Medical University

Xiuhua Guo ( $\nabla$ statguo@ccmu.edu.cn )

School of Public Health, Capital Medical University https://orcid.org/0000-0001-6657-6940 
Research

Keywords: Cerebrovascular diseases, Type 2 diabetes, Comorbidities, Gaseous pollutants, Distributed lag nonlinear model

Posted Date: April 5th, 2021

DOl: https://doi.org/10.21203/rs.3.rs-384411/v1

License: (c) (i) This work is licensed under a Creative Commons Attribution 4.0 International License.

Read Full License 
1 Short-term effects of gaseous pollutants on cause-specific

2 cerebrovascular disease among patients with type 2 diabetes: case-

3 crossover evidence from Beijing, China

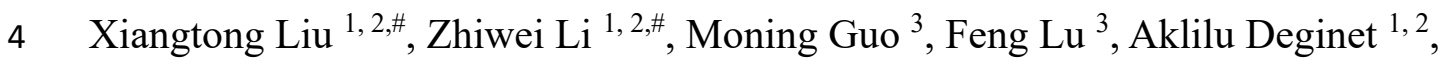

5 Mengmeng Liu ${ }^{1,2}$, Xiaolin $\mathrm{Xu}^{4,5}$, Yanxia Luo ${ }^{1,2}$, Yaoyu $\mathrm{Hu}^{1,2}$, Yutong $\mathrm{Li}^{1,2}$,

6 Mengqiu Wu ${ }^{1,2}$, Mengyang Liu ${ }^{1,2}$, Lixin Tao ${ }^{1,2, *}$, Xiuhua Guo ${ }^{1,2, *}$

$7{ }^{1}$ School of Public Health, Capital Medical University, Beijing, 100069, China.

$8 \quad 2$ Beijing Municipal Key Laboratory of Clinical Epidemiology, Beijing, 100069, China.

$9 \quad{ }^{3}$ Beijing Municipal Health Commission Information Center, Beijing, 100034, China.

$10{ }^{4}$ The University of Queensland, Brisbane, Australia.

115 School of Public Health, Zhejiang University, Hangzhou, 310058, China.

12 \#Xiangtong Liu and Zhiwei Li contributed equally to the work.

13 Corresponding authors:

14 Xiuhua Guo

15 School of Public Health, Capital Medical University, No.10 Xitoutiao, You'anmenWai,

16 Fengtai District, Beijing 100069, China

Fax: +86-010-83911508, Tel.: +86-010-83911508

E-mail: $\underline{\text { statguo@,ccmu.edu.cn }}$

19 Lixin Tao

School of Public Health, Capital Medical University, No.10 Xitoutiao, You'anmenWai,

Fengtai District, Beijing 100069, China

Fax: +86-010-83911778, Tel.: +86-010-83911778

E-mail: taolixin@.ccmu.edu.cn

E-mail Address

Xiangtong Liu Ph.D

E-mail Address: xiangtongl@ccmu.edu.cn

Zhiwei Li Ph.D 
E-mail Address: 15128472546@163.com

29

Moning Guo MD

E-mail Address: 13581627289@163.com

Feng Lu Ph.D

E-mail Address: lufeng@wjw.beijng.gov.cn

Aklilu Deginet Ph.D

E-mail Address: dgaklilu2006@gmail.com

Mengmeng Liu MD

E-mail Address: liumengmeng@ccmu.edu.cn

Xiaolin Xu Ph.D

E-mail Address: xiaolin.xu@uq.edu.au

Yanxia Luo Ph.D

E-mail Address: lyx100@ccmu.edu.cn

Yaoyu Hu MS

E-mail Address: huyaoyu499@163.com

Yutong Li MS

E-mail Address: 15210355177@163.com

Mengqiu Wu MS

E-mail Address: qiuwm00@163.com

Mengyang Liu Ph.D

E-mail Address: 18031864301@163.com

Lixin Tao Ph.D

E-mail Address: taolixin@ccmu.edu.cn

Xiuhua Guo Ph.D

E-mail Address: statguo@ccmu.edu.cn

Word count of abstract: 280

Word count of text: 4836 


\section{Highlights}

56

57

58

59

60

61

62

63

64
- The health effect of gaseous pollutants pollution on cerebrovascular morbidity among the patients with comorbid type 2 diabetes in developing countries has remained largely unknown.

- Exposure to gaseous pollutants is associated an increased risk of cerebrovascular morbidity among the patient with comorbid type 2 diabetes.

$\mathrm{NO}_{2}, \mathrm{SO}_{2}, \mathrm{O}_{3}$ and $\mathrm{CO}$ presented non-linear and lagged effect on cerebrovascular disease among patients with type 2 diabetes, which may vary by gender and age.

- The study provided evidence of the risk of comorbid type 2 diabetes and cerebrovascular disease due to gaseous pollutants. 


\section{Abstract}

Background: The association between gaseous pollutants and cerebrovascular morbidity has been widely studied; however, the association in patients with type 2 diabetes has remained unknown in developing countries.

Methods: A time-stratified case-crossover design combined with a distributed lag nonlinear model was adopted to estimate the short-term effects of gaseous pollutants (nitrogen dioxide $\left[\mathrm{NO}_{2}\right]$, sulfur dioxide $\left[\mathrm{SO}_{2}\right]$, ozone $\left[\mathrm{O}_{3}\right]$, and carbon monoxide $[\mathrm{CO}]$ ) on cerebrovascular cases among patients with type 2 diabetes in Beijing. In addition, our study explored the variability across sex and age groups.

Results: A total of 223,216 (male $57.6 \%$ and elderly $61.7 \%$ ) cerebrovascular cases were reported from 2014 to 2018 . The cumulative exposure-response curves were Ushaped for $\mathrm{NO}_{2}$, J-shaped for $\mathrm{SO}_{2}$, and $\mathrm{V}$-shaped for $\mathrm{O}_{3}$ and $\mathrm{CO}$. Extreme low-O3, low$\mathrm{CO}$ and high-CO increased the risk of cerebrovascular morbidity, with the maximum relative risk (RR) of 1.14 (95\% CI: 1.01-1.28) (1st vs median), 1.02 (95\% CI: 1.01 1.03) (1st vs median) and 1.25 (95\% CI: 1.09-1.45) (99th vs median), respectively, appearing at lag0-13. Elderly individuals aged over 65 years were susceptible to extremely low- $\mathrm{O}_{3}$ (maximum RR: $1.27,95 \%$ CI: 1.08-1.48). Men and elderly individuals were more susceptible to extremely high-CO, and the maximum RR was $10 \%[1.31(1.11-1.54)$ vs. $1.19(1.00,1.41)]$ and $26 \%$ higher $[1.41(1.17-1.69)$ vs. 1.12 $(0.97,1.30)]$ than women and adults, respectively.

Conclusions: $\mathrm{NO}_{2}, \mathrm{SO}_{2}, \mathrm{O}_{3}$ and $\mathrm{CO}$ presented nonlinear and lagged effects on cerebrovascular disease among patients with type 2 diabetes, which may vary by sex and age. Our study added to the limited evidence for the risk of cerebrovascular disease due to gaseous pollutants among patients with comorbid type 2 diabetes.

Keywords: Cerebrovascular diseases; Type 2 diabetes; Comorbidities; Gaseous pollutants; Distributed lag nonlinear model 


\section{Introduction}

Cerebrovascular disease remains a global challenge to public health and is the leading cause of mortality (Feigin et al., 2017). The cerebrovascular disease burden has increased in China (Wang et al., 2017), accounting for approximately one-quarter of the overall mortality (Zhou et al., 2016). One-third of cerebrovascular cases are comorbid with type 2 diabetes (T2D), leading to poor prognosis (Buddeke et al., 2017). To reduce the disease burden of comorbidity of cerebrovascular disease and $\mathrm{T} 2 \mathrm{D}$, it is crucial to determine modifiable risk factors.

Gaseous pollutants are common determinants for both T2D and cerebrovascular disease (Paul et al., 2020; Tian et al., 2018). Existing studies have proven that nitrogen dioxide $\left(\mathrm{NO}_{2}\right)$ is an important modifiable risk factor for cerebrovascular disease (Byrne et al., 2020; Maheswaran et al., 2016). Recently, the impact of gaseous pollutants on diabetes are attracting attention (Byrne et al., 2020; Sui et al., 2020). However, few studies have focused on the influence of gaseous pollutants on the incidence of cerebrovascular disease among people with comorbidities in the real world. Chiusolo et al. (Chiusolo et al., 2011) found that patients with preexisting diabetes appeared to confer a stronger susceptibility to $\mathrm{NO}_{2}$ exposure. A recent study suggested that individuals with comorbidities, especially diabetes, tend to be more susceptible to air pollution (Li et al., 2019).

The disease burden of comorbidities has become a serious issue for public health in China. However, the short-term effects of ambient gaseous pollutant exposure on cerebrovascular disease onset among diabetic participants in China remain largely unknown. Additionally, these associations at the city-specific level have not been thoroughly investigated, particularly in Beijing.

In this study, we aimed to examine the associations between ambient gaseous 
pollutants $\left(\mathrm{NO}_{2}\right.$, sulfur dioxide $\left[\mathrm{SO}_{2}\right]$, ozone $\left[\mathrm{O}_{3}\right]$ and carbon monoxide $\left.[\mathrm{CO}]\right)$ and overall cerebrovascular disease onset among patients with comorbid T2D in Beijing. In addition, subgroups analysis of sex and age were adopted to explore the associations in susceptible subpopulations.

\section{Materials and Methods}

\subsection{Study population}

The data on cerebrovascular cases among patients with T2D during Jan 1st 2014 and Dec 31st 2018 were extracted from the Beijing Municipal Health Commission Information Center (http://www.phic.org.cn/). The data recording system in the study area has been proven to be of high validity and good representativeness, covering more than 95\% of medical services for permanent residents in Beijing (Aklilu et al., 2020). The geographic locations of 258 hospitals included in the analysis have been described in our previous study (Li et al., 2018).

Data capture from the medical record system, including patient age, sex, date of admission, T2D status, and principal diagnosis on discharge. Based on the principal diagnosis, cause-specific cerebrovascular cases were identified according to the International Classification of Diseases, $10^{\text {th }}$ Revision (ICD-10) codes: hemorrhagic stroke (ICD-10: I60-I62), ischemic stroke (ICD-10: I63), atherosclerosis (ICD-10: I65), intracranial aneurysm (ICD-10: I67) and apoplexy sequela (ICD-10: I69). Admissions caused by unintentional injuries or surgeries were excluded according to the principal diagnosis. In the study, overall cerebrovascular admissions were calculated as the sum of hemorrhagic stroke, ischemic stroke, atherosclerosis, intracranial aneurysm and apoplexy sequelae. A history of T2D was coded as E12 and was defined as having fasting plasma glucose $\geq 7.1 \mathrm{mmol} / \mathrm{L}$ and/or current treatment of diabetes with antidiabetic medication before admission. According to the living address of cases, only 
cerebrovascular disease admissions among residents living in Beijing were included in this analysis (Figure S1).

\subsection{Air pollution and meteorological data}

Daily data of $\mathrm{NO}_{2}, \mathrm{SO}_{2}, \mathrm{O}_{3}, \mathrm{CO}$, fine particulate matter $\left(\mathrm{PM}_{2.5}\right)$ and inhalable particulate matter $\left(\mathrm{PM}_{10}\right)$, from Jan 1, 2014 to Dec 31, 2018, were obtained acquired from the Beijing Environmental Protection Bureau (http://www.bjepb.gov.cn/). Average daily temperature and relative humidity were acquired from the China Meteorological Data Sharing Service System (https://data.cma.cn/en).

\subsection{Ethical Clearance}

In this study, data were collected as part of health surveillance mandated by government and all data were desensitized. Since we only analyzed the aggregated data, which were typically used for administrative purposes, informed consent was waived. However, the study was approved by the Institutional Review Board of Capital Medical University with the IRB00009511 identification number.

\subsection{Statistical Analysis}

A time-stratified case-crossover design was adopted to remove control confounders and long-term trends (Liu et al., 2021). A distributed lag nonlinear model (DLNM) was applied to estimate the nonlinear and lagged influence of gaseous pollutants on cerebrovascular disease onset. Based on bidimensional functions called "cross-basis", DLNM can reflect both lag structure of the association and the nonlinear exposure responses (Gasparrini, 2014). Therefore, the combination of the case-crossover design with DLNM was applied to simultaneously estimate the short-term, nonlinear, and lagged effects of gaseous pollutants on cerebrovascular disease onset.

In our study, a DLNM model combined with a time-stratified case-crossover design was built as follows: 


$$
Y_{t} \sim \operatorname{Poisson}\left(\mu_{t}\right) \log \left(\mu_{t}\right)
$$$$
=\alpha+c b\left(\text { Pollutant }_{i, t}, 3\right)+P C A_{i}+c b\left(\text { Temp }_{t}, 3\right)+c b\left(R H_{t}, 3\right)
$$$$
+\gamma \text { Holiday }_{t}+\lambda \text { Stratum }
$$

where $t$ is the specific day of observation; $Y_{t}$ is the number of cerebrovascular cases on $t ; \mu_{t}$ is the expectation of $Y_{t} ; \alpha$ is the intercept; Pollutant ${ }_{i, t}$, Temp $_{t}$, and $R H_{t}$ are, respectively, the $i^{\text {th }}$ concentration of gaseous pollutant, daily temperature and relative humidity on $t ; c b()$ is the cross-basis function for exposure-response natural spline for gaseous pollutant, daily temperature or relative humidity. Holiday is the indicator variable used to control the effect of public holidays, and Stratum represents the time stratum of the case-crossover design.

Sensitivity analyses were applied to check the robustness of the effect. First, a natural spline function of $3 d f$ was added to the model (Chen et al., 2018). Second, principal component analysis was adopted to estimate the independent effects of gaseous pollutant (Feng et al., 2019). Principal component analysis is an appropriate statistical method to deal with collinearity among pollutants (Stafoggia et al., 2017). For example, to estimate the independent effects of $\mathrm{NO}_{2}$, we first substituted all primary pollutants $\left(\mathrm{SO}_{2}, \mathrm{O}_{3}, \mathrm{CO}, \mathrm{PM}_{2.5}\right.$ and $\left.\mathrm{PM}_{10}\right)$ with a composite latent variable, and then included this latent variable in the DLNM model. Additionally, the coefficient $\beta^{\prime}$ was transformed into the coefficient $\beta$ of the original pollutants.

The 3D, slice and cumulative exposure-response curves were plotted between gaseous pollutants and cerebrovascular morbidity among patients with T2D. To identify the effect of extreme concentrations of gaseous pollutants, relative risk (RR) was estimated by comparing cerebrovascular cases of the 99th above or 1st below percentiles to the median concentration. We estimated the cumulative lag influence (lag $0-1$, lag $0-3$, lag $0-5$, lag $0-7$, lag $0-9$, lag $0-11$ and lag $0-13$ ) to effectively describe the 
associations between gaseous pollutants and cerebrovascular cases. Subgroup analyses were performed to test the variability across sex and age groups (adult: 18-64 years; elderly individuals: $\geq 65$ years).

All analyses were conducted in R software (version 4.0.2) and dlnm package was adopted for fitting the DLNM.

\section{Results}

A total of 223,216 cases for cerebrovascular disease were collected after excluding nonlocal admissions, of which 9,541 (4.27\%), 149,757 (67.09\%), 7,444 (3.33\%), $31,356(14.05 \%)$, and $23,912(10.71 \%)$ were hospitalized for hemorrhagic stroke, ischemic stroke, atherosclerosis, intracranial aneurysm, and apoplexy sequela, respectively. In short, male participants and elderly individuals ( $\geq 65$ years) accounted for $57.6 \%$ and $61.7 \%$, respectively. Table 1 summarizes the characteristics and distribution of daily cerebrovascular disease (overall and cause-specific), stratified by sex and age over the study period.

During the period, the 1st, 50th (median) and 99th percentiles of concentration, respectively, were $14.32,43.66,112.79\left(\mu \mathrm{g} / \mathrm{m}^{3}\right)$ for $\mathrm{NO}_{2}, 2.2,7.35,74.51\left(\mu \mathrm{g} / \mathrm{m}^{3}\right)$ for $\mathrm{SO}_{2}, 5.85,54.91,157.06\left(\mu \mathrm{g} / \mathrm{m}^{3}\right)$ for $\mathrm{O}_{3}$, and $0.28,0.89,4.84\left(\mathrm{mg} / \mathrm{m}^{3}\right)$ for $\mathrm{CO}$. The average concentrations of $\mathrm{NO}_{2}, \mathrm{SO}_{2}, \mathrm{O}_{3}$ and $\mathrm{CO}$ were $1.1,0.1,0.3$ and 0.2 times of the limit of Chinese Ambient Air Quality guideline II, respectively. The differences in $\mathrm{NO}_{2}$, $\mathrm{SO}_{2}, \mathrm{O}_{3}$ and $\mathrm{CO}$ between the four seasons were statistically significant $\left(\mathrm{F}_{\mathrm{NO}_{2}}=76.62\right.$, $\left.P<0.001 ; \mathrm{FSO}_{2}=188.1, P<0.001 ; \mathrm{FO}_{3}=559.5, P<0.001 ; \mathrm{FCO}_{\mathrm{CO}}=117.2, P<0.001\right)$. During the study period, the median daily temperature was $14.4^{\circ} \mathrm{C}$ and the mean relative humidity was $54.3 \pm 19.2 \%$. Gaseous pollutants except $\mathrm{O}_{3}$ were strongly correlated with each other (coefficients ranged from 0.49 to 0.87 ) and moderately correlated with relative humidity and temperature (coefficients ranged from -0.41 to 0.33 ) (Fig. 1). 
216 Table 1. Characteristics and distribution of daily cerebrovascular cases in Beijing (2014-2018).

\begin{tabular}{|c|c|c|c|c|c|c|c|c|c|c|}
\hline & \multicolumn{2}{|c|}{ Total } & \multicolumn{2}{|l|}{ Male } & \multicolumn{2}{|c|}{ Female } & \multicolumn{2}{|c|}{$<65 \mathrm{y}$} & \multicolumn{2}{|l|}{$\geq 65 \mathrm{y}$} \\
\hline & $\mathrm{N}$ & $\mathrm{P}_{25}-\mathrm{P}_{75}$ & N (\%) & $\mathrm{P}_{25}-\mathrm{P}_{75}$ & N (\%) & $\mathrm{P}_{25}-\mathrm{P}_{75}$ & $\mathrm{~N}(\%)$ & $\mathrm{P}_{25}-\mathrm{P}_{75}$ & $\mathrm{~N}(\%)$ & $\mathrm{P}_{25}-\mathrm{P}_{75}$ \\
\hline Hemorrhagic stroke & 9541 & $3-7$ & $5692(59.7)$ & $2-4$ & $3848(40.3)$ & $1-3$ & $4218(44.7)$ & $1-3$ & $5323(55.3)$ & $2-4$ \\
\hline Ischemic stroke & 149757 & $52-104$ & $87285(58.2)$ & $30-61$ & $62469(41.8)$ & $23-44$ & $57454(38.2)$ & $21-40$ & $92303(61.8)$ & $31-65$ \\
\hline Atherosclerosis & 7444 & $1-6$ & $5460(73.2)$ & $1-4$ & $1984(26.8)$ & $0-2$ & $3412(45.3)$ & $0-3$ & $4032(54.7)$ & $1-3$ \\
\hline Intracranial aneurysm & 31356 & $11-22$ & $14876(47.4)$ & $5-11$ & $16480(52.6)$ & $6-12$ & $13068(41.6)$ & $4-10$ & $18288(58.4)$ & $6-13$ \\
\hline Apoplexy sequela & 23912 & $3-21$ & $14550(61.0)$ & $2-13$ & $9361(39.0)$ & $1-8$ & $7049(30.5)$ & $1-6$ & $16863(69.5)$ & $2-15$ \\
\hline Overall & 223216 & $72-157$ & $128508(57.6)$ & $41-91$ & 94703 (42.4) & $32-66$ & $85726(38.3)$ & $30-60$ & $137490(61.7)$ & $43-98$ \\
\hline
\end{tabular}

217 Note: N-counts of cases; $\mathrm{P}_{25}-\mathrm{P}_{75}$ : 25th percentile and 75 th percentile of daily cases. 


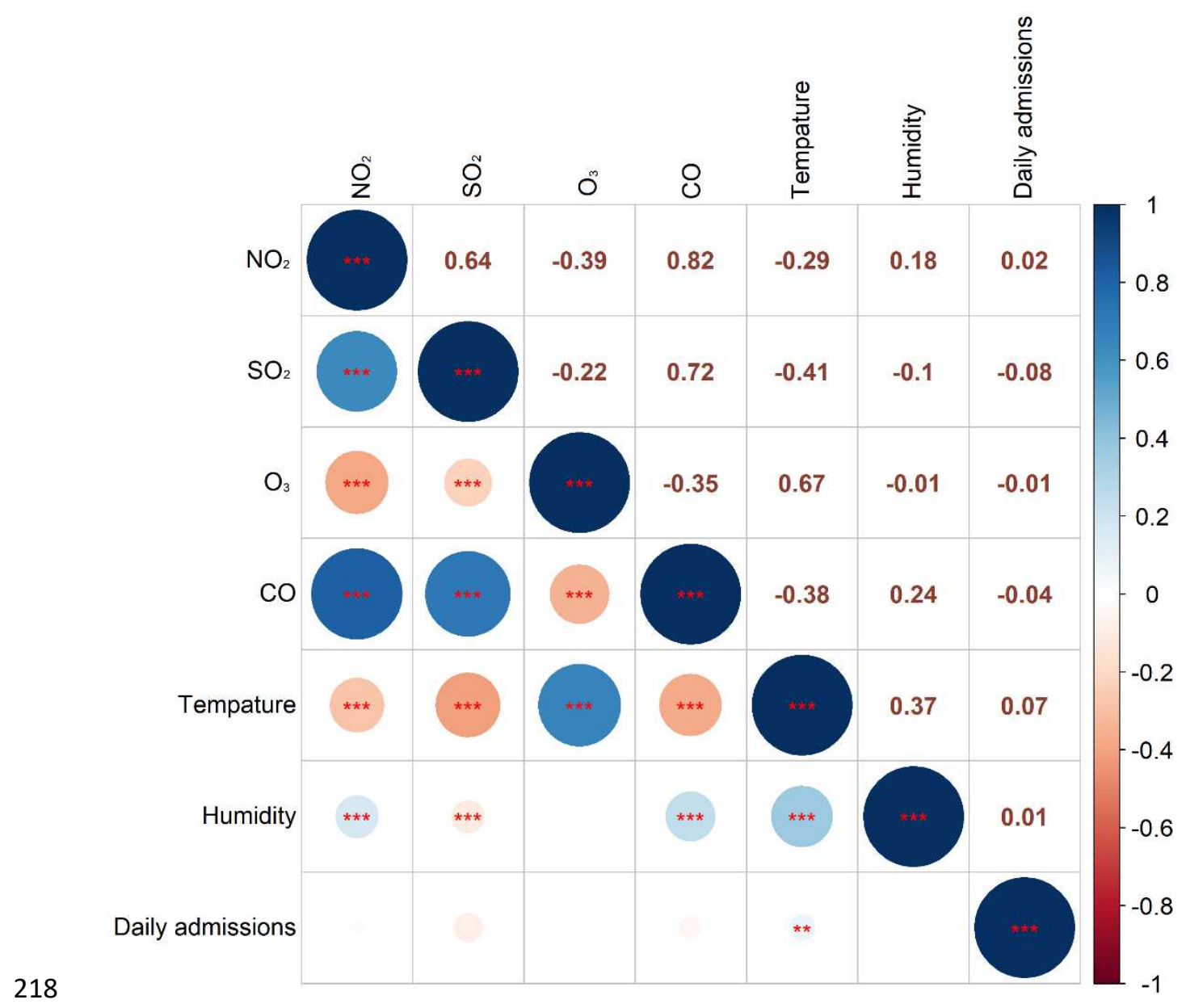

219 Fig.1 Pearson correlation coefficients between gaseous air pollutants concentrations and weather conditions and daily admissions for cerebrovascular disease in Beijing, 2014-2018 $(\mathbf{n}=\mathbf{1 7 9 9})$. $\mathrm{NO}_{2}$ : nitrogen dioxide; $\mathrm{SO}_{2}$ : sulfur dioxide;

$\mathrm{O}_{3}$ : ozone; CO: carbon monoxide. $* 0.01<P \leq 0.05 ; * * 0.001<P \leq 0.01 ; * * * P \leq 0.001$.

The results of the multipollutant models indicated that $\mathrm{SO}_{2}, \mathrm{NO}_{2}, \mathrm{O}_{3}$ and $\mathrm{CO}$ were significantly associated with both overall and cause-specific cerebrovascular morbidity. Figure 2 show the relative risks of each gaseous pollutant on overall and cause-specific cerebrovascular morbidity using different lag structures. The maximum relative risk 227 (RR) was 1.004 (95\% CI: 1.003-1.004) for cerebrovascular cases, 1.003 (95\% CI: 1.002-1.004) for ischemic stroke, 1.006 (95\% CI: 1.003-1.009) for atherosclerosis, 1.005 (95\% CI: 1.003-1.006) for intracranial aneurysm, and 1.007 (95\% CI: $1.005-$ 1.009) for apoplexy sequela. High $\mathrm{SO}_{2}$ concentrations were associated with an 231 increased risk of overall cerebrovascular cases (RR: 1.001; 95\% CI: 1.0003-1.0017) at 
(a) $\mathrm{SO}_{2}$

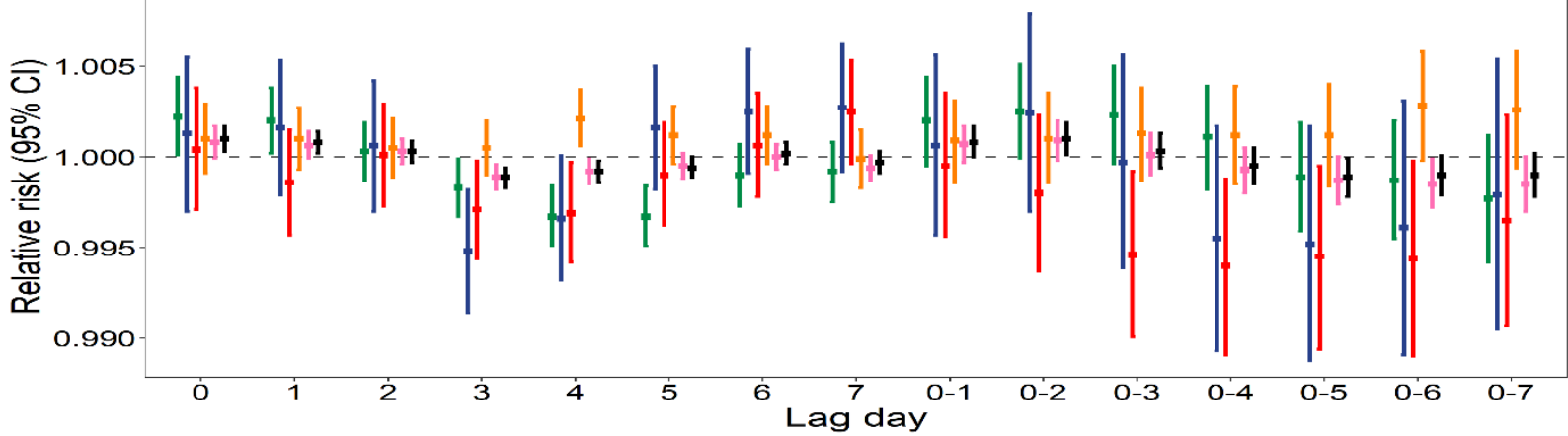

(b) $\mathrm{NO}_{2}$

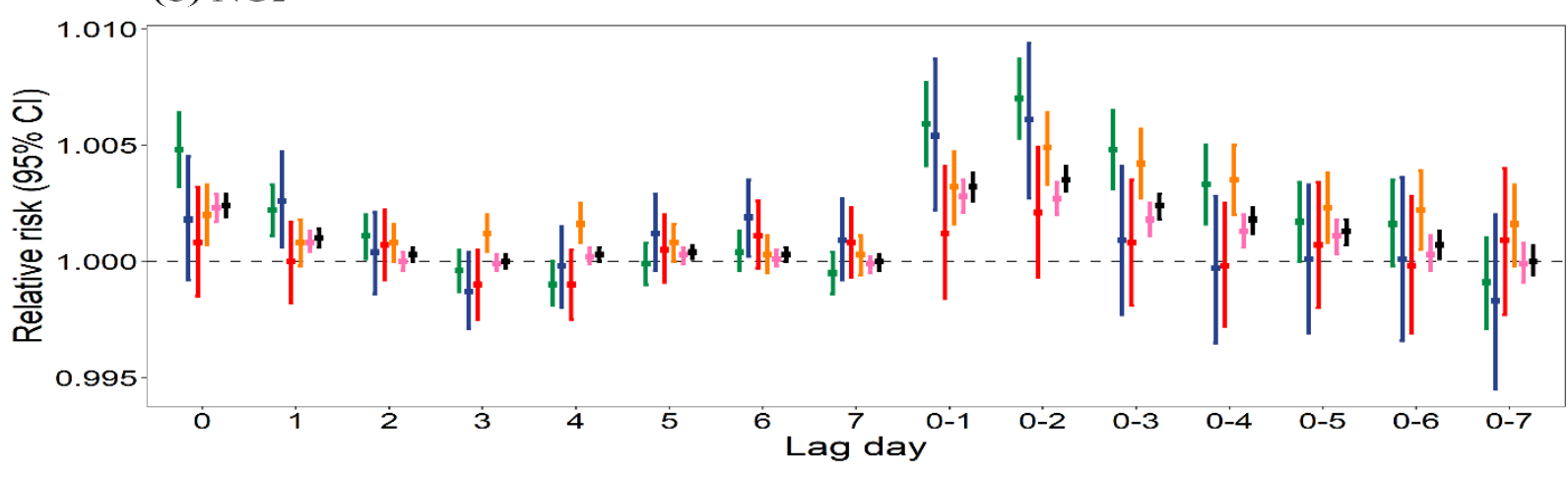

(c) $\mathrm{O}_{3}$

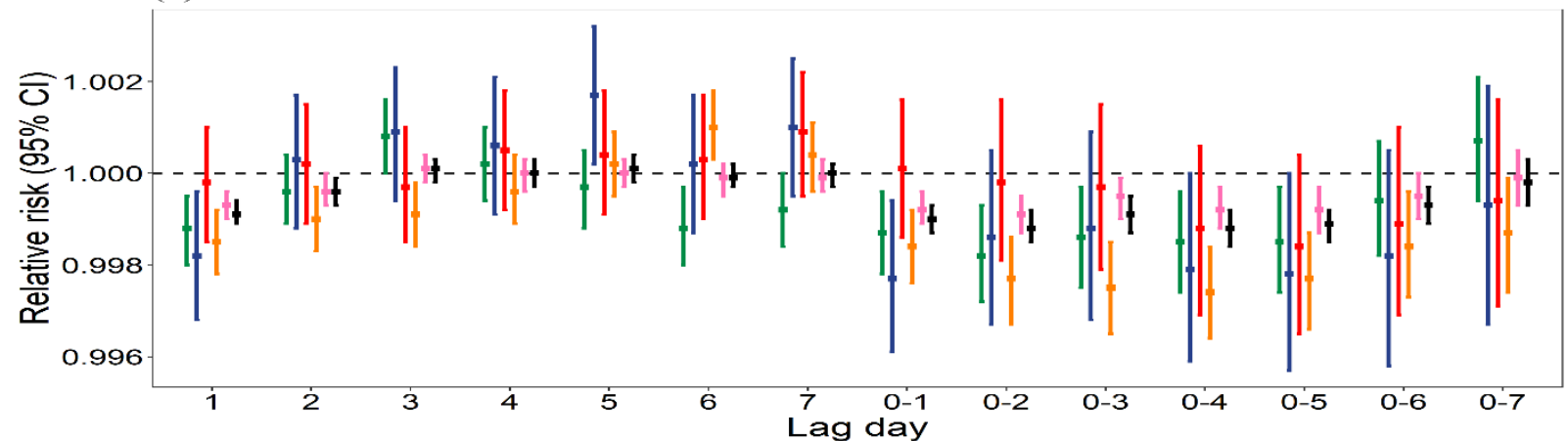

(d) $\mathrm{CO}$

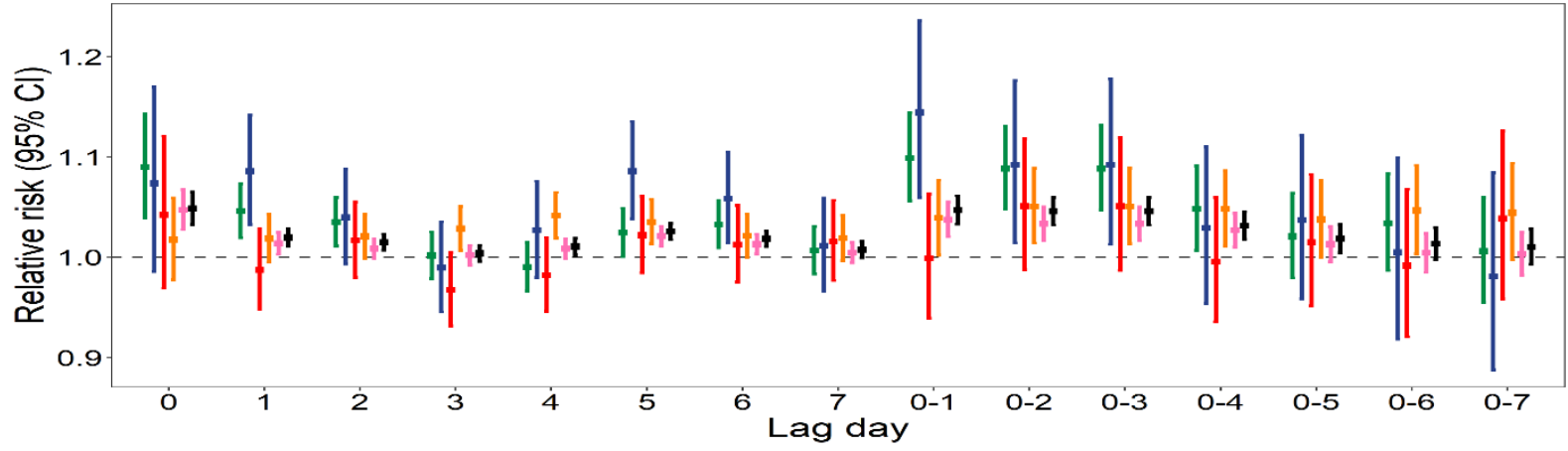

Fig.2 Relative risk with $95 \%$ CI (confidence interval) in cerebrovascular morbidity associated with increase in concentrations of gaseous pollutants for different lag structures in multi-pollutant model: (a) $\mathrm{SO}_{2}$ : sulfur dioxide; (b) $\mathrm{NO}_{2}$ : nitrogen dioxide; (c) $\mathrm{O}_{3}$ : ozone; (d) $\mathrm{CO}$ : carbon monoxide. 
lag0 day, intracranial aneurysm cases (RR: 1.0021; 95\% CI: 1.0006-1.0037) at lag4 day, and apoplexy sequela cases (RR: 1.0022; 95\% CI: 1.0001-1.0044) at lag0 day (Fig. 2a). Positive associations were observed between $\mathrm{NO}_{2}$ concentrations and risk for overall and cause specific cerebrovascular cases except hemorrhagic stroke (Fig. 2b).

High $\mathrm{O}_{3}$ concentrations were associated with an increased risk of overall cerebrovascular cases (RR: 1.0101; 95\% CI: 1.0099-1.0104) at lag0 day, hemorrhagic stroke cases (RR: 1.0386; 95\% CI: 1.0373-1.040) at lag0 day, ischemic stroke cases (RR: $1.003 ; 95 \%$ CI: 1.0026-1.0033) at lag0 day, atherosclerosis cases (RR: $1.0017 ; 95 \%$ CI: 1.0002-1.0032) at lag5 day, and intracranial aneurysm cases (RR: 1.0441; 95\% CI: 1.0434-1.0449) at lag0 day (Fig. 2c). High CO concentrations were associated with increased risk for overall cerebrovascular cases and ischemic stroke, atherosclerosis, intracranial aneurysm, and apoplexy sequela case morbidity, and the maximum RR and 95\% CI were 1.048 (95\% CI: 1.032-1.065), 1.047 (95\% CI: 1.027-1.067), 1.144 (95\% CI: $1.060-1.236), 1.050$ (95\% CI: 1.014-1.088), and 1.099 (95\% CI: 1.056-1.144), respectively (Fig. 2d).

Figure 3 presents the 3-D exposure-response surfaces of $\mathrm{NO}_{2}, \mathrm{SO}_{2}, \mathrm{O}_{3}$ and $\mathrm{CO}$ on overall cerebrovascular cases along lag days, which indicated that the associations tended to be nonlinear. As shown in Figure 4, the cumulative exposure-response curves of the overall association between gaseous pollutants and cerebrovascular cases among T2D patients were almost nonlinear. Generally, the cumulative exposure-response curves for $\mathrm{NO}_{2}$ were almost U-shaped, with a slight decline at concentrations lower than $43 \mu \mathrm{g} / \mathrm{m}^{3}$ and then a flattening off. Evidently, J-shaped increased risks were observed for high $\mathrm{SO}_{2}$ concentrations, with steep slopes for concentrations $\geq 10 \mu \mathrm{g} / \mathrm{m}^{3}$ and becoming flat at $45 \mu \mathrm{g} / \mathrm{m}^{3}$. The cumulative exposure-response curves for $\mathrm{O}_{3}$ were V-shaped, which meant that both high and low concentrations of $\mathrm{O}_{3}$ may increase the 
risk of cerebrovascular disease onset among patients with T2D. In addition, the

263 decreased.

(a) Overall effect of $\mathrm{NO}_{2}$ on CBVD

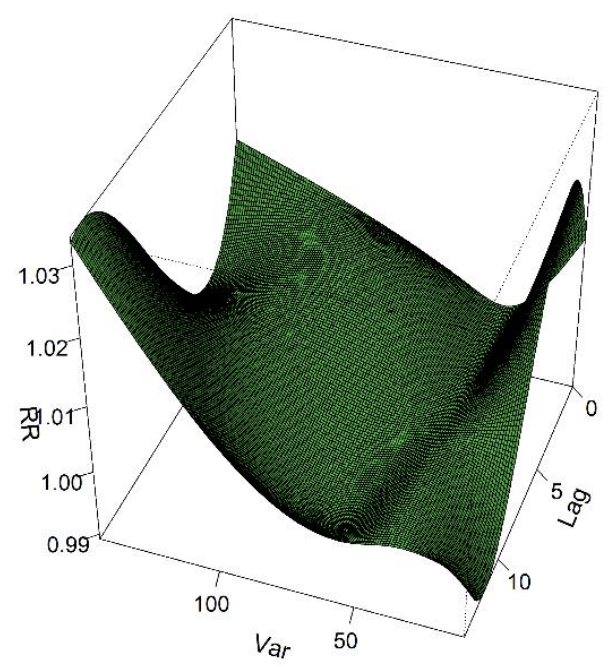

(c) Overall effect of $\mathrm{O}_{3}$ on $\mathrm{CBVD}$

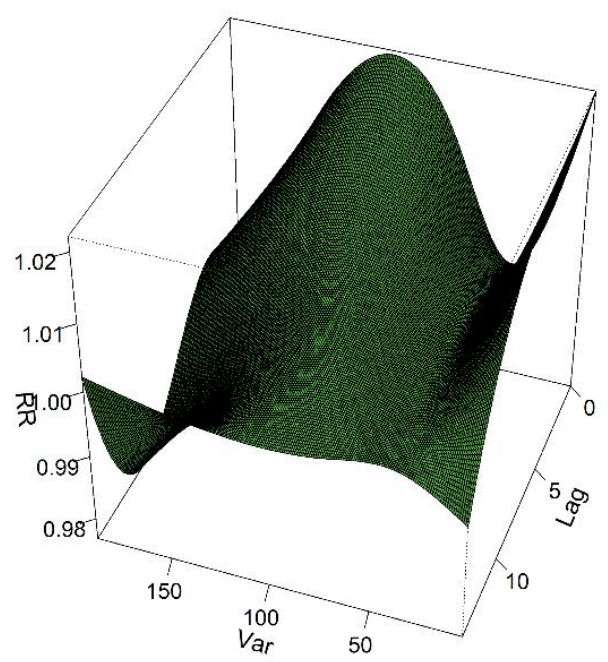

(b) Overall effect of $\mathrm{sO}_{2}$ on CBVD

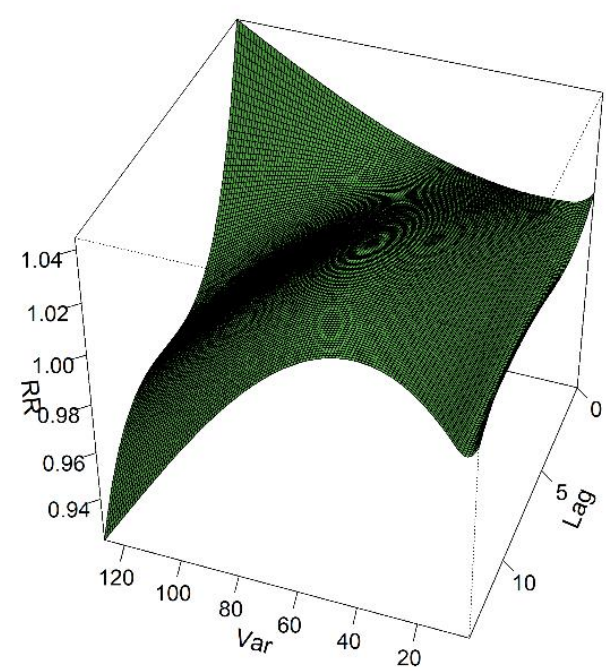

(d) Overall effect of CO on CBVD

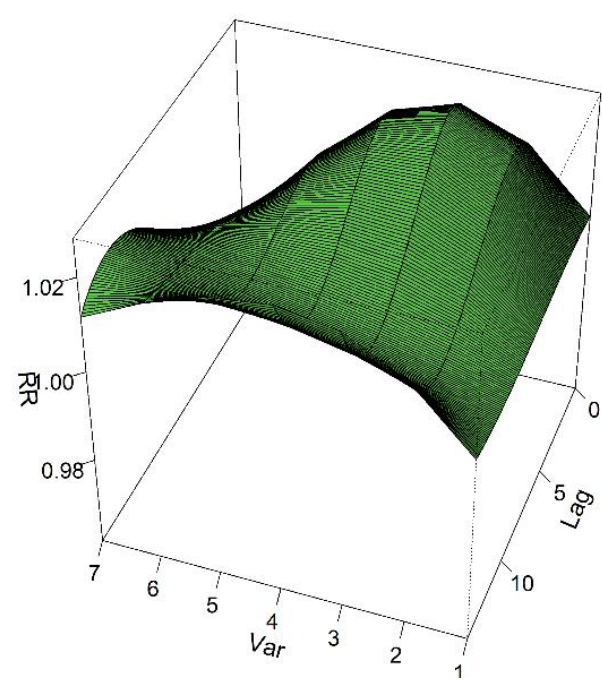

Fig.3 3D graphs of $\mathrm{NO}_{2}, \mathrm{SO}_{2}, \mathrm{O}_{3}$ and $\mathrm{CO}$ effect on overall cerebrovascular cases:

(a) overall effect of $\mathrm{NO}_{2}$; (b) overall effect of $\mathrm{SO}_{2}$; (c) overall effect of $\mathrm{O}_{3}$; (d) overall effect of $\mathrm{CO}$. $\mathrm{NO}_{2}$ : nitrogen dioxide; $\mathrm{SO}_{2}$ : sulfur dioxide; $\mathrm{O}_{3}$ : ozone; $\mathrm{CO}$ : carbon monoxide. RR (95\% CI): relative risk and 95\% confidence interval. 
(a) Cumulative effect of $\mathrm{NO}_{2}$

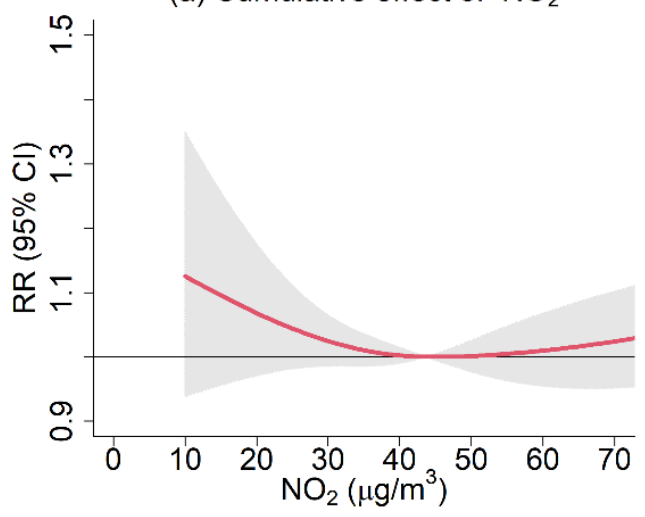

(c) Cumulative effect of $\mathrm{O}_{3}$

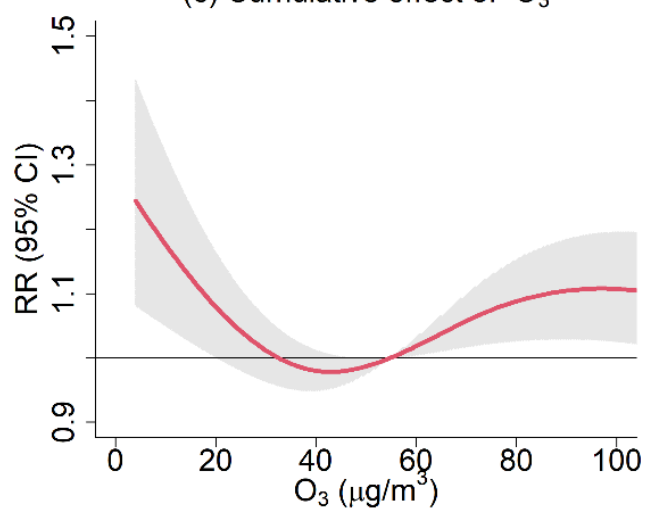

(b) Cumulative effect of $\mathrm{SO}_{2}$

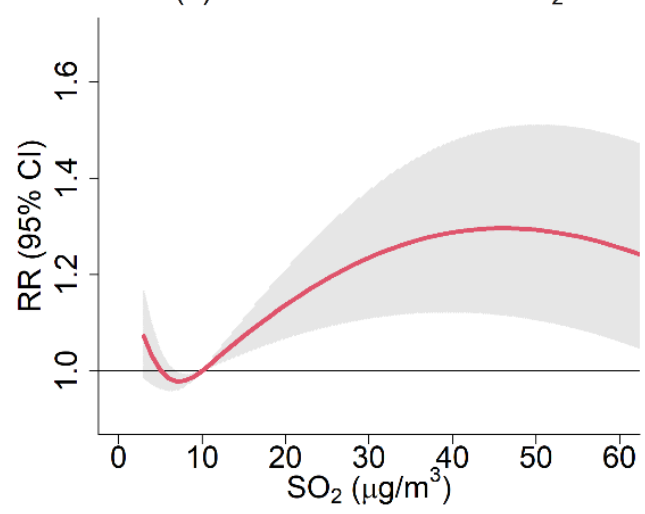

(d) Cumulative effect of $\mathrm{CO}$

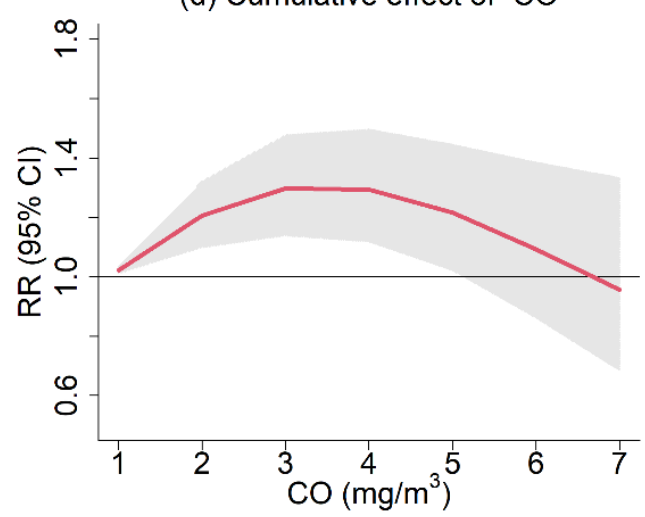

Fig.4 Cumulative exposure-response curves on overall cerebrovascular cases for gaseous pollutants $\left(\mathrm{NO}_{2}, \mathrm{SO}_{2}, \mathrm{O}_{3}\right.$ and $\left.\mathrm{CO}\right)$ at lag0-7 using multi-pollutant models in Beijing, 2014-2018: (a) cumulative effect of $\mathrm{NO}_{2}$; (b) cumulative effect of $\mathrm{SO}_{2}$; (c) cumulative effect of $\mathrm{O}_{3}$; (d) cumulative effect of $\mathrm{CO}$. $\mathrm{NO}_{2}$ : nitrogen dioxide; $\mathrm{SO}_{2}$ : sulfur dioxide; $\mathrm{O}_{3}$ : ozone; $\mathrm{CO}$ : carbon monoxide. $\mathrm{RR}(95 \% \mathrm{CI})$ : relative risk and $95 \%$ confidence interval.

The single day lag-response curves for extreme gaseous pollutant concentrations in cerebrovascular cases are shown in Figure 5. As shown in the figure, the curves of extremely high- $\mathrm{NO}_{2}$, high- $\mathrm{O}_{3}$ and high- $\mathrm{SO}_{2}$ were almost straight lines through lag days, and no statistically significant associations were observed. The extremely highCO showed a statistically significant hazardous effect from lag 2 to lag 7 , and the RR value peaked at lag 4 . The curves of the extremely low- $\mathrm{NO}_{2}$, low- $\mathrm{SO}_{2}$, and low- $\mathrm{CO}$ were S-shaped, and the RR values peaked at lag 3, lag 10, and lag 4, respectively. The extremely low-CO presented statistically significant hazardous effect from lag2 to lag7. 

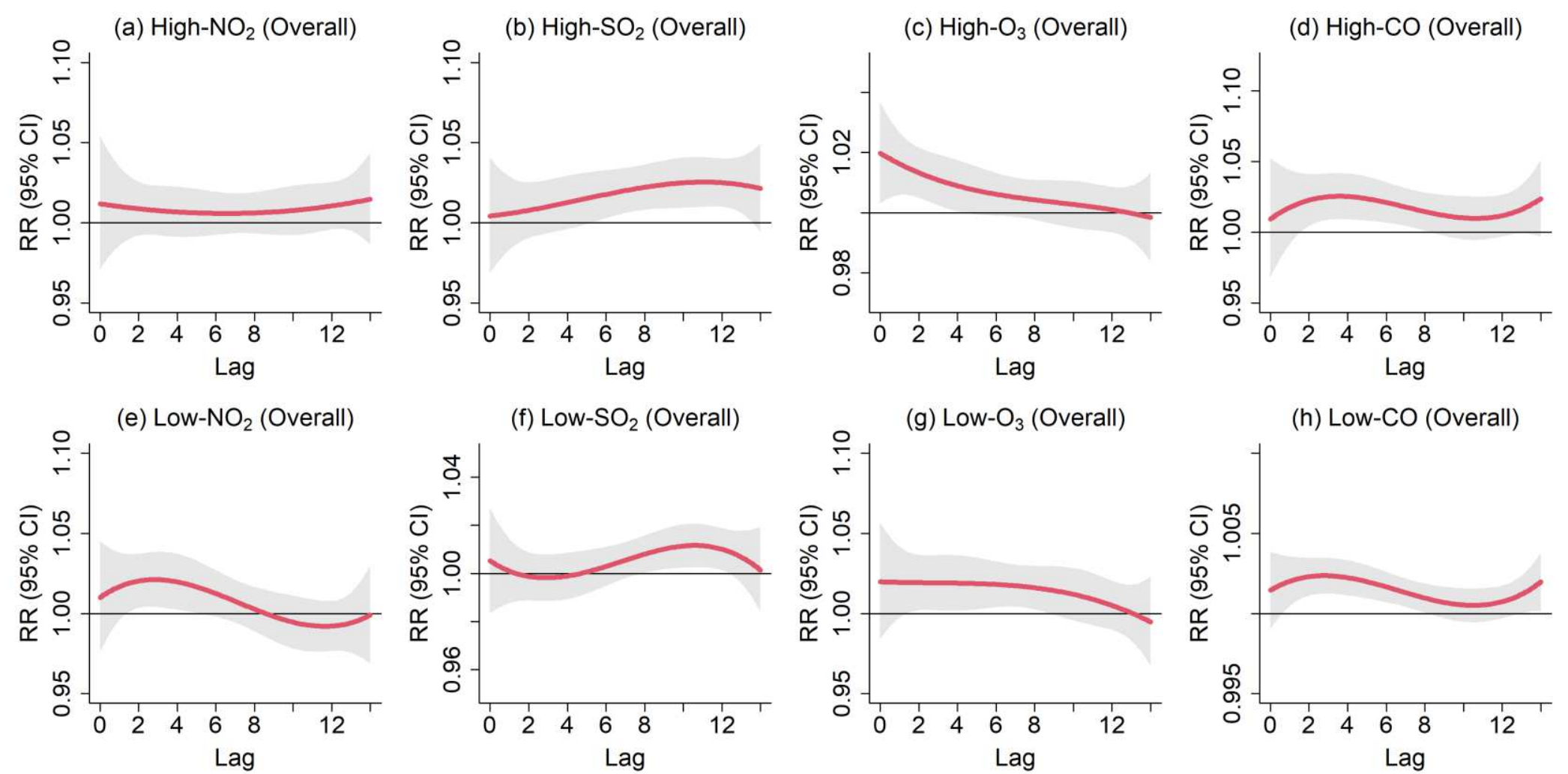

Fig.5 Single day lag-response curves on overall cerebrovascular cases for extreme gaseous pollutants concentrations using multi-pollutant model in Beijing, 2014-2018: (a) overall effect of extreme high- $\mathrm{NO}_{2}$; (b) overall effect of extreme high- $\mathrm{SO}_{2}$; (c) overall effect of extreme high-O 3 ; (d) overall effect of extreme high-CO; (e) overall effect of extreme low- $\mathrm{NO}_{2}$; (f) overall effect of extreme low-SO $\mathrm{O}_{3}$; (h) overall effect of extreme low-CO. The high effect was estimated by the RR of cerebrovascular disease by comparing the 99th percentile to the median of daily concentration, whereas the low effect was estimated by comparing the 1 st percentile to the median of daily concentration. $\mathrm{NO}_{2}$ : nitrogen dioxide; $\mathrm{SO}_{2}$ : sulfur dioxide; $\mathrm{O}_{3}$ : ozone; $\mathrm{CO}$ : carbon monoxide. $\mathrm{RR}(95 \% \mathrm{CI})$ : relative risk and $95 \%$ confidence interval. 
Table 2 lists the cumulative effect of extreme concentrations of gaseous pollutants on cerebrovascular disease among patients with T2D at different lag days. In short, extremely low- $\mathrm{O}_{3}$, low- $\mathrm{CO}$ and high- $\mathrm{CO}$ presented statistically significant hazardous cumulative effects. However, no prominent cumulative effect of extreme $\mathrm{NO}_{2}$ and $\mathrm{SO}_{2}$ was observed. Specifically, low $\mathrm{O}_{3}$ was significantly associated with an increased risk for cerebrovascular disease ( $\mathrm{RR}=1.14,95 \% \mathrm{CI}: 1.01-1.28)$ (1st vs median) at lag0-13. Low- $\mathrm{CO}$ had a slight adverse effect ( $\mathrm{RR}=1.02,95 \% \mathrm{CI}: 1.01-1.03)$ (1st vs median) at lag0-13. Extremely high CO presented a statistically significant cumulative hazardous effect on cerebrovascular cases, which increased with extended lag days, with a maximum RR value of 1.25 (95\% CI: 1.09-1.45) (99th vs median), appearing at lag013. Differences were also observed among subgroups of the population. Subgroup analysis indicated that extremely low- $\mathrm{O}_{3}$ only had a significant cumulative effect in the elderly population, with a maximum RR value of 1.27 (95\% CI: $1.08-1.48)$ (1st vs median) on lag 0-13. Men and elderly individuals were more susceptible to extreme cumulative high-CO than women and adults, and the maximum RR value was $10 \%$ (1.31 (95\% CI: $1.11-1.54)$ vs. $1.19(95 \%$ CI: $1.00,1.41))$ and $26 \%$ higher $(1.41(95 \%$ CI: $1.17-1.69)$ vs. $1.12(95 \%$ CI: $0.97,1.30))$, respectively. 
Table 2 Cumulative RRs and 95\% CIs of extreme gaseous pollutants concentrations on cerebrovascular cases using multi-pollutant models.

\begin{tabular}{|c|c|c|c|c|c|c|c|c|c|c|c|c|c|c|c|}
\hline \multirow[b]{2}{*}{ Pollutant } & \multirow[b]{2}{*}{ Population } & \multicolumn{7}{|c|}{ Extreme-low effect } & \multicolumn{7}{|c|}{ Extreme-high effect } \\
\hline & & $\operatorname{lag} 0-1$ & $\operatorname{lag} 0-3$ & $\operatorname{lag} 0-5$ & $\operatorname{lag} 0-7$ & lag0-9 & $\begin{array}{r}\text { lag } \\
0-11\end{array}$ & $\begin{array}{r}\text { lag } \\
0-13\end{array}$ & $\operatorname{lag} 0-1$ & $\operatorname{lag} 0-3$ & $\operatorname{lag} 0-5$ & $\operatorname{lag} 0-7$ & $\operatorname{lag} 0-9$ & $\begin{array}{r}\text { lag } \\
0-11\end{array}$ & $\begin{array}{r}\text { lag } \\
0-13\end{array}$ \\
\hline $\mathrm{NO}_{2}$ & Total & $\begin{array}{c}0.99 \\
(0.97 \\
1.02)\end{array}$ & $\begin{array}{r}1.01 \\
(0.97 \\
1.05)\end{array}$ & $\begin{array}{c}1.03 \\
(0.99 \\
1.08)\end{array}$ & $\begin{array}{c}1.04 \\
(0.98 \\
1.09)\end{array}$ & $\begin{array}{c}1.03 \\
(0.97 \\
1.10)\end{array}$ & $\begin{array}{r}1.03 \\
(0.96 \\
1.10)\end{array}$ & $\begin{array}{r}1.03 \\
(0.96 \\
1.10)\end{array}$ & $\begin{array}{c}1.03 \\
(0.96 \\
1.10)\end{array}$ & $\begin{array}{c}1.05 \\
(0.95 \\
1.17)\end{array}$ & $\begin{array}{c}1.05 \\
(0.94 \\
1.18)\end{array}$ & $\begin{array}{c}1.03 \\
(0.91 \\
1.17)\end{array}$ & $\begin{array}{c}1.02 \\
(0.89 \\
1.17)\end{array}$ & $\begin{array}{c}1.03 \\
(0.88 \\
1.20)\end{array}$ & $\begin{array}{c}1.06 \\
(0.89 \\
1.25)\end{array}$ \\
\hline & Men & $\begin{array}{r}1.00 \\
(0.98 \\
1.03)\end{array}$ & $\begin{array}{c}1.03 \\
(0.99 \\
1.07)\end{array}$ & $\begin{array}{c}1.05 \\
(1.00 \\
1.11)\end{array}$ & $\begin{array}{c}1.06 \\
(1.00, \\
1.13)\end{array}$ & $\begin{array}{c}1.06 \\
(0.99 \\
1.13)\end{array}$ & $\begin{array}{r}1.05 \\
(0.97 \\
1.13)\end{array}$ & $\begin{array}{r}1.05 \\
(0.97 \\
1.14)\end{array}$ & $\begin{array}{c}1.04 \\
(0.96 \\
1.12)\end{array}$ & $\begin{array}{r}1.06 \\
(0.95 \\
1.20)\end{array}$ & $\begin{array}{r}1.06 \\
(0.92 \\
1.21)\end{array}$ & $\begin{array}{r}1.04 \\
(0.90 \\
1.20)\end{array}$ & $\begin{array}{c}1.03 \\
(0.87 \\
1.21)\end{array}$ & $\begin{array}{r}1.04 \\
(0.87 \\
1.25)\end{array}$ & $\begin{array}{r}1.09 \\
(0.89 \\
1.32)\end{array}$ \\
\hline & Women & $\begin{array}{r}0.98 \\
(0.95 \\
1.01)\end{array}$ & $\begin{array}{r}0.98 \\
(0.94 \\
1.03)\end{array}$ & $\begin{array}{r}1.00 \\
(0.95 \\
1.06)\end{array}$ & $\begin{array}{r}1.01 \\
(0.94 \\
1.07)\end{array}$ & $\begin{array}{c}1.00 \\
(0.93 \\
1.07)\end{array}$ & $\begin{array}{r}1.00 \\
(0.92 \\
1.08)\end{array}$ & $\begin{array}{r}1.00 \\
(0.92 \\
1.09)\end{array}$ & $\begin{array}{r}1.01 \\
(0.94 \\
1.1)\end{array}$ & $\begin{array}{c}1.04 \\
(0.92 \\
1.17)\end{array}$ & $\begin{array}{c}1.04 \\
(0.91 \\
1.19)\end{array}$ & $\begin{array}{c}1.03 \\
(0.88 \\
1.19)\end{array}$ & $\begin{array}{r}1.01 \\
(0.85 \\
1.20)\end{array}$ & $\begin{array}{r}1.01 \\
(0.84 \\
1.21)\end{array}$ & $\begin{array}{r}1.02 \\
(0.84 \\
1.25)\end{array}$ \\
\hline & Adult & $\begin{array}{r}1.00 \\
(0.97 \\
1.03)\end{array}$ & $\begin{array}{r}1.02 \\
(0.97 \\
1.07)\end{array}$ & $\begin{array}{c}1.03 \\
(0.97 \\
1.10)\end{array}$ & $\begin{array}{r}1.04 \\
(0.97, \\
1.12)\end{array}$ & $\begin{array}{r}1.04 \\
(0.96 \\
1.13)\end{array}$ & $\begin{array}{r}1.04 \\
(0.95 \\
1.14)\end{array}$ & $\begin{array}{r}1.04 \\
(0.94, \\
1.14)\end{array}$ & $\begin{array}{r}1.05 \\
(0.96 \\
1.15)\end{array}$ & $\begin{array}{r}1.07 \\
(0.93 \\
1.23)\end{array}$ & $\begin{array}{r}1.06 \\
(0.91 \\
1.24)\end{array}$ & $\begin{array}{r}1.07 \\
(0.91 \\
1.27)\end{array}$ & $\begin{array}{c}1.09 \\
(0.91 \\
1.32)\end{array}$ & $\begin{array}{r}1.11 \\
(0.90 \\
1.37)\end{array}$ & $\begin{array}{r}1.12 \\
(0.90, \\
1.41)\end{array}$ \\
\hline & $\begin{array}{l}\text { The } \\
\text { elderly }\end{array}$ & $\begin{array}{c}0.99 \\
(0.96 \\
1.01)\end{array}$ & $\begin{array}{r}1.00 \\
(0.97 \\
1.04)\end{array}$ & $\begin{array}{c}1.03 \\
(0.98 \\
1.08)\end{array}$ & $\begin{array}{r}1.03 \\
(0.98 \\
1.09)\end{array}$ & $\begin{array}{c}1.03 \\
(0.96 \\
1.09)\end{array}$ & $\begin{array}{r}1.02 \\
(0.95 \\
1.09)\end{array}$ & $\begin{array}{r}1.02 \\
(0.95 \\
1.10)\end{array}$ & $\begin{array}{c}1.01 \\
(0.94 \\
1.08)\end{array}$ & $\begin{array}{r}1.04 \\
(0.93 \\
1.16)\end{array}$ & $\begin{array}{c}1.04 \\
(0.92 \\
1.18)\end{array}$ & $\begin{array}{r}1.01 \\
(0.88 \\
1.15)\end{array}$ & $\begin{array}{c}0.97 \\
(0.84 \\
1.13)\end{array}$ & $\begin{array}{r}0.97 \\
(0.83 \\
1.15)\end{array}$ & $\begin{array}{r}1.02 \\
(0.85 \\
1.22)\end{array}$ \\
\hline $\mathrm{SO}_{2}$ & Total & $\begin{array}{r}0.99 \\
(0.98 \\
1.01)\end{array}$ & $\begin{array}{r}1.00 \\
(0.97 \\
1.02)\end{array}$ & $\begin{array}{r}1.00 \\
(0.97 \\
1.03)\end{array}$ & $\begin{array}{r}0.99 \\
(0.96 \\
1.03)\end{array}$ & $\begin{array}{c}0.99 \\
(0.95 \\
1.03)\end{array}$ & $\begin{array}{r}1.00 \\
(0.96 \\
1.05)\end{array}$ & $\begin{array}{c}1.03 \\
(0.98, \\
1.08)\end{array}$ & $\begin{array}{r}1.02 \\
(0.96 \\
1.09)\end{array}$ & $\begin{array}{r}1.05 \\
(0.94 \\
1.16)\end{array}$ & $\begin{array}{c}1.06 \\
(0.93 \\
1.20)\end{array}$ & $\begin{array}{c}1.07 \\
(0.93 \\
1.24)\end{array}$ & $\begin{array}{r}1.09 \\
(0.92 \\
1.28)\end{array}$ & $\begin{array}{r}1.10 \\
(0.92 \\
1.32)\end{array}$ & $\begin{array}{c}1.12 \\
(0.93, \\
1.36)\end{array}$ \\
\hline
\end{tabular}




\begin{tabular}{|c|c|c|c|c|c|c|c|c|c|c|c|c|c|c|c|}
\hline & Men & $\begin{array}{r}0.99 \\
(0.97 \\
1.01)\end{array}$ & $\begin{array}{r}1.00 \\
(0.97 \\
1.03)\end{array}$ & $\begin{array}{r}1.01 \\
(0.97 \\
1.05)\end{array}$ & $\begin{array}{r}1.01 \\
(0.97 \\
1.05)\end{array}$ & $\begin{array}{r}1.01 \\
(0.96 \\
1.06)\end{array}$ & $\begin{array}{r}1.02 \\
(0.97 \\
1.07)\end{array}$ & $\begin{array}{r}1.04 \\
(0.98 \\
1.10)\end{array}$ & $\begin{array}{r}1.03 \\
(0.96 \\
1.11)\end{array}$ & $\begin{array}{r}1.06 \\
(0.94 \\
1.20)\end{array}$ & $\begin{array}{r}1.07 \\
(0.92 \\
1.24)\end{array}$ & $\begin{array}{r}1.08 \\
(0.91 \\
1.28)\end{array}$ & $\begin{array}{r}1.08 \\
(0.90 \\
1.31)\end{array}$ & $\begin{array}{r}1.08 \\
(0.88 \\
1.33)\end{array}$ & $\begin{array}{r}1.10 \\
(0.88 \\
1.37)\end{array}$ \\
\hline & Women & $\begin{array}{r}0.99 \\
(0.97 \\
1.01)\end{array}$ & $\begin{array}{r}0.99 \\
(0.96 \\
1.02)\end{array}$ & $\begin{array}{r}0.99 \\
(0.95 \\
1.03)\end{array}$ & $\begin{array}{r}0.98 \\
(0.93 \\
1.02)\end{array}$ & $\begin{array}{r}0.97 \\
(0.92 \\
1.02)\end{array}$ & $\begin{array}{r}0.98 \\
(0.93 \\
1.04)\end{array}$ & $\begin{array}{r}1.01 \\
(0.95 \\
1.07)\end{array}$ & $\begin{array}{r}1.01 \\
(0.94 \\
1.09)\end{array}$ & $\begin{array}{r}1.03 \\
(0.91 \\
1.16)\end{array}$ & $\begin{array}{r}1.04 \\
(0.89, \\
1.21)\end{array}$ & $\begin{array}{r}1.06 \\
(0.89 \\
1.26)\end{array}$ & $\begin{array}{r}1.09 \\
(0.90 \\
1.32)\end{array}$ & $\begin{array}{r}1.13 \\
(0.92 \\
1.39)\end{array}$ & $\begin{array}{r}1.16 \\
(0.93 \\
1.46)\end{array}$ \\
\hline & Adult & $\begin{array}{r}1.00 \\
(0.98 \\
1.02)\end{array}$ & $\begin{array}{r}1.00 \\
(0.97, \\
1.04)\end{array}$ & $\begin{array}{r}1.00 \\
(0.96 \\
1.04)\end{array}$ & $\begin{array}{r}0.99 \\
(0.94 \\
1.04)\end{array}$ & $\begin{array}{r}0.99 \\
(0.93 \\
1.04)\end{array}$ & $\begin{array}{r}1.01 \\
(0.95 \\
1.07)\end{array}$ & $\begin{array}{r}1.04 \\
(0.98 \\
1.12)\end{array}$ & $\begin{array}{r}1.01 \\
(0.93 \\
1.10)\end{array}$ & $\begin{array}{r}1.00 \\
(0.87 \\
1.15)\end{array}$ & $\begin{array}{r}0.99 \\
(0.84 \\
1.18)\end{array}$ & $\begin{array}{r}1.02 \\
(0.84 \\
1.23)\end{array}$ & $\begin{array}{r}1.05 \\
(0.84 \\
1.30)\end{array}$ & $\begin{array}{r}1.07 \\
(0.85 \\
1.36)\end{array}$ & $\begin{array}{r}1.08 \\
(0.84 \\
1.39)\end{array}$ \\
\hline & $\begin{array}{l}\text { The } \\
\text { elderly }\end{array}$ & $\begin{array}{r}0.99 \\
(0.97, \\
1.01)\end{array}$ & $\begin{array}{r}0.99 \\
(0.97 \\
1.02)\end{array}$ & $\begin{array}{r}1.00 \\
(0.97 \\
1.04)\end{array}$ & $\begin{array}{r}1.00 \\
(0.96 \\
1.04)\end{array}$ & $\begin{array}{r}0.99 \\
(0.95 \\
1.04)\end{array}$ & $\begin{array}{r}1.00 \\
(0.95 \\
1.05)\end{array}$ & $\begin{array}{r}1.02 \\
(0.96 \\
1.07)\end{array}$ & $\begin{array}{r}1.03 \\
(0.97 \\
1.11)\end{array}$ & $\begin{array}{r}1.08 \\
(0.96 \\
1.21)\end{array}$ & $\begin{array}{r}1.10 \\
(0.96 \\
1.26)\end{array}$ & $\begin{array}{r}1.11 \\
(0.95 \\
1.29)\end{array}$ & $\begin{array}{r}1.11 \\
(0.93 \\
1.32)\end{array}$ & $\begin{array}{r}1.12 \\
(0.93 \\
1.36)\end{array}$ & $\begin{array}{r}1.15 \\
(0.94 \\
1.41)\end{array}$ \\
\hline $\mathrm{O}_{3}$ & Total & $\begin{array}{r}1.02 \\
(0.98 \\
1.05)\end{array}$ & $\begin{array}{r}1.05 \\
(0.98 \\
1.12)\end{array}$ & $\begin{array}{r}1.08 \\
(0.99 \\
1.17)\end{array}$ & $\begin{array}{r}1.09 \\
(1.00 \\
1.20)\end{array}$ & $\begin{array}{r}1.10 \\
(1.00 \\
1.22)\end{array}$ & $\begin{array}{r}1.12 \\
(1.00 \\
1.25)\end{array}$ & $\begin{array}{r}1.14 \\
(1.01 \\
1.28)\end{array}$ & $\begin{array}{r}1.04 \\
(0.98 \\
1.09)\end{array}$ & $\begin{array}{r}1.05 \\
(0.97 \\
1.13)\end{array}$ & $\begin{array}{r}1.04 \\
(0.95 \\
1.15)\end{array}$ & $\begin{array}{r}1.04 \\
(0.93 \\
1.17)\end{array}$ & $\begin{array}{r}1.04 \\
(0.92 \\
1.19)\end{array}$ & $\begin{array}{r}1.03 \\
(0.89 \\
1.19)\end{array}$ & $\begin{array}{r}1.00 \\
(0.85 \\
1.17)\end{array}$ \\
\hline & Men & $\begin{array}{r}1.01 \\
(0.97 \\
1.06)\end{array}$ & $\begin{array}{r}1.04 \\
(0.97 \\
1.12)\end{array}$ & $\begin{array}{r}1.07 \\
(0.97 \\
1.17)\end{array}$ & $\begin{array}{r}1.08 \\
(0.97 \\
1.21)\end{array}$ & $\begin{array}{r}1.10 \\
(0.97 \\
1.23)\end{array}$ & $\begin{array}{r}1.12 \\
(0.98 \\
1.27)\end{array}$ & $\begin{array}{r}1.15 \\
(1.00 \\
1.32)\end{array}$ & $\begin{array}{r}1.04 \\
(0.98 \\
1.10)\end{array}$ & $\begin{array}{r}1.06 \\
(0.97 \\
1.16)\end{array}$ & $\begin{array}{r}1.05 \\
(0.94 \\
1.17)\end{array}$ & $\begin{array}{r}1.02 \\
(0.90 \\
1.16)\end{array}$ & $\begin{array}{r}0.99 \\
(0.85 \\
1.15)\end{array}$ & $\begin{array}{r}0.96 \\
(0.81 \\
1.14)\end{array}$ & $\begin{array}{r}0.95 \\
(0.79 \\
1.13)\end{array}$ \\
\hline & Women & $\begin{array}{r}1.02 \\
(0.98 \\
1.07)\end{array}$ & $\begin{array}{r}1.06 \\
(0.98 \\
1.14)\end{array}$ & $\begin{array}{r}1.09 \\
(0.99 \\
1.20)\end{array}$ & $\begin{array}{r}1.10 \\
(0.99 \\
1.23)\end{array}$ & $\begin{array}{r}1.12 \\
(0.99 \\
1.26)\end{array}$ & $\begin{array}{r}1.12 \\
(0.98 \\
1.28)\end{array}$ & $\begin{array}{r}1.13 \\
(0.98 \\
1.30)\end{array}$ & $\begin{array}{r}1.04 \\
(0.98 \\
1.10)\end{array}$ & $\begin{array}{r}1.03 \\
(0.94 \\
1.13)\end{array}$ & $\begin{array}{r}1.03 \\
(0.92 \\
1.16)\end{array}$ & $\begin{array}{r}1.08 \\
(0.94 \\
1.23)\end{array}$ & $\begin{array}{r}1.12 \\
(0.96 \\
1.31)\end{array}$ & $\begin{array}{r}1.12 \\
(0.94 \\
1.33)\end{array}$ & $\begin{array}{r}1.07 \\
(0.89 \\
1.29)\end{array}$ \\
\hline
\end{tabular}




\begin{tabular}{|c|c|c|c|c|c|c|c|c|c|c|c|c|c|c|c|}
\hline & Adult & $\begin{array}{r}1.01 \\
(0.97 \\
1.05)\end{array}$ & $\begin{array}{r}1.03 \\
(0.97 \\
1.11)\end{array}$ & $\begin{array}{r}1.05 \\
(0.96 \\
1.14)\end{array}$ & $\begin{array}{r}1.05 \\
(0.95 \\
1.16)\end{array}$ & $\begin{array}{r}1.05 \\
(0.94 \\
1.17)\end{array}$ & $\begin{array}{r}1.06 \\
(0.94 \\
1.19)\end{array}$ & $\begin{array}{r}1.06 \\
(0.94 \\
1.21)\end{array}$ & $\begin{array}{r}1.05 \\
(1.00, \\
1.11)\end{array}$ & $\begin{array}{r}1.06 \\
(0.98 \\
1.15)\end{array}$ & $\begin{array}{r}1.03 \\
(0.93 \\
1.14)\end{array}$ & $\begin{array}{r}1.01 \\
(0.89, \\
1.14)\end{array}$ & $\begin{array}{r}1.01 \\
(0.88 \\
1.16)\end{array}$ & $\begin{array}{r}1.00 \\
(0.86 \\
1.17)\end{array}$ & $\begin{array}{r}0.99 \\
(0.84 \\
1.17)\end{array}$ \\
\hline & $\begin{array}{l}\text { The } \\
\text { elderly }\end{array}$ & $\begin{array}{r}1.02 \\
(0.97 \\
1.08)\end{array}$ & $\begin{array}{c}1.08 \\
(0.99 \\
1.17)\end{array}$ & $\begin{array}{c}1.12 \\
(1.01 \\
1.25)\end{array}$ & $\begin{array}{r}1.16 \\
(1.03 \\
1.31)\end{array}$ & $\begin{array}{r}1.19 \\
(1.04, \\
1.37)\end{array}$ & $\begin{array}{r}1.23 \\
(1.06, \\
1.43)\end{array}$ & $\begin{array}{r}1.27 \\
(1.08 \\
1.48)\end{array}$ & $\begin{array}{r}1.01 \\
(0.94 \\
1.08)\end{array}$ & $\begin{array}{c}1.03 \\
(0.93 \\
1.14)\end{array}$ & $\begin{array}{r}1.06 \\
(0.94 \\
1.21)\end{array}$ & $\begin{array}{r}1.10 \\
(0.95 \\
1.28)\end{array}$ & $\begin{array}{r}1.10 \\
(0.93 \\
1.32)\end{array}$ & $\begin{array}{r}1.06 \\
(0.88 \\
1.29)\end{array}$ & $\begin{array}{r}1.01 \\
(0.82 \\
1.25)\end{array}$ \\
\hline \multirow[t]{5}{*}{$\mathrm{CO}$} & Total & $\begin{array}{r}1.00 \\
(1.00 \\
1.01)\end{array}$ & $\begin{array}{r}1.01 \\
(1.00 \\
1.01)\end{array}$ & $\begin{array}{c}1.01 \\
(1.00 \\
1.02)\end{array}$ & $\begin{array}{r}1.01 \\
(1.01, \\
1.02)\end{array}$ & $\begin{array}{c}1.02 \\
(1.01, \\
1.02)\end{array}$ & $\begin{array}{r}1.02 \\
(1.01, \\
1.03)\end{array}$ & $\begin{array}{r}1.02 \\
(1.01, \\
1.03)\end{array}$ & $\begin{array}{r}1.04 \\
(0.98 \\
1.10)\end{array}$ & $\begin{array}{c}1.08 \\
(0.99 \\
1.18)\end{array}$ & $\begin{array}{r}1.11 \\
(1.01 \\
1.23)\end{array}$ & $\begin{array}{r}1.15 \\
(1.03, \\
1.28)\end{array}$ & $\begin{array}{r}1.19 \\
(1.05 \\
1.35)\end{array}$ & $\begin{array}{c}1.22 \\
(1.07 \\
1.40)\end{array}$ & $\begin{array}{r}1.25 \\
(1.09 \\
1.45)\end{array}$ \\
\hline & Men & $\begin{array}{r}1.00 \\
(1.00 \\
1.01)\end{array}$ & $\begin{array}{r}1.01 \\
(1.00 \\
1.02)\end{array}$ & $\begin{array}{c}1.01 \\
(1.01 \\
1.02)\end{array}$ & $\begin{array}{c}1.02 \\
(1.01, \\
1.03)\end{array}$ & $\begin{array}{c}1.02 \\
(1.01, \\
1.03)\end{array}$ & $\begin{array}{r}1.02 \\
(1.01, \\
1.03)\end{array}$ & $\begin{array}{r}1.02 \\
(1.01, \\
1.03)\end{array}$ & $\begin{array}{r}1.06 \\
(0.99 \\
1.13)\end{array}$ & $\begin{array}{c}1.12 \\
(1.01, \\
1.24)\end{array}$ & $\begin{array}{r}1.15 \\
(1.02 \\
1.29)\end{array}$ & $\begin{array}{r}1.19 \\
(1.04, \\
1.35)\end{array}$ & $\begin{array}{r}1.23 \\
(1.07 \\
1.42)\end{array}$ & $\begin{array}{r}1.27 \\
(1.09 \\
1.49)\end{array}$ & $\begin{array}{r}1.31 \\
(1.11, \\
1.54)\end{array}$ \\
\hline & Women & $\begin{array}{r}1.00 \\
(1.00 \\
1.01)\end{array}$ & $\begin{array}{r}1.00 \\
(1.00, \\
1.01)\end{array}$ & $\begin{array}{c}1.01 \\
(1.00 \\
1.01)\end{array}$ & $\begin{array}{r}1.01 \\
(1.00, \\
1.02)\end{array}$ & $\begin{array}{r}1.01 \\
(1.00, \\
1.02)\end{array}$ & $\begin{array}{r}1.01 \\
(1.00 \\
1.02)\end{array}$ & $\begin{array}{r}1.01 \\
(1.00 \\
1.02)\end{array}$ & $\begin{array}{r}1.01 \\
(0.94 \\
1.08)\end{array}$ & $\begin{array}{r}1.04 \\
(0.93 \\
1.15)\end{array}$ & $\begin{array}{r}1.07 \\
(0.95 \\
1.21)\end{array}$ & $\begin{array}{r}1.10 \\
(0.97 \\
1.26)\end{array}$ & $\begin{array}{r}1.14 \\
(0.98 \\
1.32)\end{array}$ & $\begin{array}{r}1.17 \\
(0.99 \\
1.37)\end{array}$ & $\begin{array}{c}1.19 \\
(1.00, \\
1.41)\end{array}$ \\
\hline & Adult & $\begin{array}{r}1.00 \\
(1.00 \\
1.01)\end{array}$ & $\begin{array}{r}1.01 \\
(1.00, \\
1.01)\end{array}$ & $\begin{array}{c}1.01 \\
(1.00 \\
1.01)\end{array}$ & $\begin{array}{r}1.01 \\
(1.00, \\
1.02)\end{array}$ & $\begin{array}{r}1.01 \\
(1.00, \\
1.02)\end{array}$ & $\begin{array}{r}1.01 \\
(1.00 \\
1.02)\end{array}$ & $\begin{array}{r}1.02 \\
(1.00 \\
1.03)\end{array}$ & $\begin{array}{r}1.01 \\
(0.95 \\
1.08)\end{array}$ & $\begin{array}{r}1.04 \\
(0.95 \\
1.15)\end{array}$ & $\begin{array}{r}1.06 \\
(0.96 \\
1.18)\end{array}$ & $\begin{array}{r}1.08 \\
(0.96 \\
1.21)\end{array}$ & $\begin{array}{r}1.09 \\
(0.96 \\
1.25)\end{array}$ & $\begin{array}{r}1.12 \\
(0.97 \\
1.30)\end{array}$ & $\begin{array}{r}1.17 \\
(1.00, \\
1.36)\end{array}$ \\
\hline & $\begin{array}{l}\text { The } \\
\text { elderly }\end{array}$ & $\begin{array}{r}1.00 \\
(1.00, \\
1.01)\end{array}$ & $\begin{array}{r}1.01 \\
(1.00, \\
1.02)\end{array}$ & $\begin{array}{c}1.01 \\
(1.00 \\
1.02)\end{array}$ & $\begin{array}{c}1.02 \\
(1.01, \\
1.03)\end{array}$ & $\begin{array}{c}1.02 \\
(1.01, \\
1.03)\end{array}$ & $\begin{array}{c}1.02 \\
(1.01, \\
1.04)\end{array}$ & $\begin{array}{c}1.02 \\
(1.01, \\
1.04)\end{array}$ & $\begin{array}{c}1.08 \\
(1.00 \\
1.16)\end{array}$ & $\begin{array}{c}1.15 \\
(1.02 \\
1.29)\end{array}$ & $\begin{array}{r}1.20 \\
(1.05, \\
1.37)\end{array}$ & $\begin{array}{r}1.28 \\
(1.10, \\
1.48)\end{array}$ & $\begin{array}{r}1.36 \\
(1.15, \\
1.60)\end{array}$ & $\begin{array}{r}1.41 \\
(1.17, \\
1.69)\end{array}$ & $\begin{array}{r}1.41 \\
(1.16, \\
1.71)\end{array}$ \\
\hline
\end{tabular}




\section{Discussion}

314 To the best of our knowledge, this is the first study to examine the associations between gaseous pollutants and cerebrovascular case onset among patients with comorbid T2D in China. In this study, we observed significant nonlinear and lagged effects of $\mathrm{NO}_{2}$, $\mathrm{SO}_{2}, \mathrm{O}_{3}$ and $\mathrm{CO}$ on the morbidity of cerebrovascular disease among patients with T2D. The extreme effect of high and low concentrations was not consonant, depending on sex, age, and type of gaseous pollutants. Our study added to the limited evidence for the risk of comorbidities of T2D and cerebrovascular disease due to gaseous pollutants in developing countries.

The nonlinear and lagged effects of gaseous pollutants were robust when adjusting for co-pollutants using principal component analysis in multipollutant models. T2D is the leading risk factor for cerebrovascular cases and related death in Chinese adults. Note that China had the largest disease burden of T2D worldwide in 2019 (Saeedi et al., 
the nonlinear and lag effects across cities due to the different study designs and model specifications. However, these findings of associations between high $\mathrm{NO}_{2}$ concentration and cerebrovascular case onset were similar as a whole.

Our results show a V-shaped CO-cerebrovascular case association at levels below the current China air quality standard. During the study period, the average daily CO concentrations $\left(0.89 \mathrm{mg} / \mathrm{m}^{3}\right)$ across Beijing were well below the current Chinese National Ambient Air Quality guideline II $\left(4 \mathrm{mg} / \mathrm{m}^{3}\right)$; however, the CO-cerebrovascular disease association remained present despite this. Furthermore, our study suggested that both low- $\mathrm{CO}$ and high- $\mathrm{CO}$ exposure may increase the risk of cerebrovascular cases. Aligning with our study, Shah and colleagues (Shah et al., 2015) found that a 1 ppm increase in CO concentrations [relative risk $(R R)$ and 95\% $C I: 1.015(1.004,1.026)$ ] was significantly associated with morbidity or mortality for stroke. Another national study reported that increases of $1 \mathrm{mg} / \mathrm{m}^{3}$ in $\mathrm{CO}$ concentrations were associated with a $3.24 \%(95 \%$ CI: $2.05 \%-4.43 \%)$ increase in ischemic stroke morbidity; however, the associations were not statistically significant when adjusting for other pollutants (Tian et al., 2018).

The concentrations of $\mathrm{SO}_{2}\left(7.35 \mu \mathrm{g} / \mathrm{m}^{3}\right)$ and $\mathrm{O}_{3}\left(54.91 \mu \mathrm{g} / \mathrm{m}^{3}\right)$ were far below the limit of Chinese Ambient Air Quality guideline II $\left(60 \mu \mathrm{g} / \mathrm{m}^{3}\right.$ and $160 \mu \mathrm{g} / \mathrm{m}^{3}$, respectively). $\mathrm{SO}_{2}$ and $\mathrm{O}_{3}$ are gaseous pollutants mainly emitted by fuel combustion and regional transport in urban Beijing. A growing body of research has suggested that incrementally increased concentrations of $\mathrm{SO}_{2}$ and $\mathrm{O}_{3}$ exposure may increase cerebrovascular morbidity (Chen et al., 2019). Contrary to previous studies, however, in our research, we observed increased cerebrovascular cases associated with high- $\mathrm{SO}_{2}$, low- $\mathrm{O}_{3}$ and high- $\mathrm{O}_{3}$ under the air quality limit of China. Note that over 2013-2018, the air quality of Beijing had a measurable improvement, of which $\mathrm{SO}_{2}$ decreased the most, 
and $\mathrm{O}_{3}$ showed a fluctuating increase (Li et al., 2020).

Our study found that exposure to extreme $\mathrm{NO}_{2}$ and $\mathrm{CO}$ may have a stronger impact on the risk for morbidity of cerebrovascular disease than $\mathrm{SO}_{2}$ and $\mathrm{O}_{3}$. This finding is almost in accordance with another national study of 172 cities in China, which reported that $\mathrm{NO}_{2}[1.82 \%(1.45 \%-2.19 \%)], \mathrm{CO}[3.24 \%(2.05 \%-4.43 \%)], \mathrm{SO}_{2}[1.37 \%(1.05 \%-$ $1.70 \%)]$ and $\mathrm{O}_{3}[0.01 \%(-0.14 \%-0.16 \%)]$ were significantly associated with increases in hospital admissions for ischemic stroke (Tian et al., 2018). Although the potential reasons remain unknown, we suppose that traffic gaseous pollutants may have become the major sources of air pollutants in Beijing. Oxidative stress is supposed to be a key pathway for the different cardiovascular actions of $\mathrm{NO}_{2}$ and $\mathrm{CO}$ pollution (Miller, 2020). A recent study found that exposure to high- $\mathrm{O}_{3}$ was associated with decreased nitrotyrosine, a marker of the reaction product of superoxide and NO (Balmes et al., 2019).

Subgroup analysis of sex supported that male participants were more susceptible to extremely high- CO exposure than females. A study from Shenzhen showed that males were vulnerable to extremely high- $\mathrm{SO}_{2}$, high- $\mathrm{NO}_{2}$ and low- $\mathrm{O}_{3}$, and females were vulnerable to extremely high- $\mathrm{O}_{3}$ (Wang et al., 2020). Another study found that among stroke patients male were more vulnerable than female to changes in concentrations of $\mathrm{SO}_{2}$ and $\mathrm{NO}_{2}$ (Wang et al., 2016). In short, the potential sex differences in the associations between gaseous pollutants and cerebrovascular cases remain to be determined.

Subgroup analysis of age showed that elder individuals were more susceptible to extremely high- $\mathrm{CO}$ and low- $\mathrm{O}_{3}$ exposure and were at higher risk of cerebrovascularrelated hospitalizations. Unsurprisingly, compared with younger adults, elderly participants are more vulnerable to gaseous pollutants and have a higher risk of 
cerebrovascular diseases. Wang and colleagues found that younger adults were more immune to extremely low- $\mathrm{NO}_{2}$ than elderly adults (Wang et al., 2020). Another study of Beijing also supported that increased concentrations of $\mathrm{NO}_{2}$ and $\mathrm{O}_{3}$ were associated with higher increased risks of cerebrovascular hospitalizations in elder individuals (Huang et al., 2017).

There are some strengths of our study. First, the data on cerebrovascular cases has been proven to be of high validity and good representativeness, covering more than $95 \%$ of permanent residents in Beijing, and the sample size was relatively large. Therefore, the present results may be representative of the authentic associations between gaseous pollution and cerebrovascular cases in the study area. Second, to the best of our knowledge, this was the first time that a nonlinear and lagged association were observed between $\mathrm{NO}_{2}, \mathrm{SO}_{2}$, and $\mathrm{O}_{3}$ and cerebrovascular cases in a population with $\mathrm{T} 2 \mathrm{D}$, which may have important implications for the prevention and treatment of cerebrovascular cases.

However, several limitations should be addressed. First, we could not collect data on the relationships between patients owing to the limitation of the available data. However, there might be few family aggregation cases, which may affect population sensitivity (Yang et al., 2018). Second, the date of diagnosis, rather than the time of cerebrovascular symptom onset, was used in the analysis, which may result in temporal misalignment between gaseous pollution and cerebrovascular incidence and the underestimation of exposure effects (Lokken et al., 2009). However, because cerebrovascular disease is a kind of disease requiring prompt hospitalization and treatment, temporal misalignment is expected to play a minor role in our estimation. As admission or diagnosis may occur in the days after the onset of symptoms, it is important to explain the lag influence of air pollution on cerebrovascular disease onset. 
Third, we used the arithmetic mean from 35 fixed-site monitor measurements as a proxy for individual exposure, which may result in exposure measurement errors and underestimate the influence of gaseous pollution (Dionisio et al., 2016). Fourth, caution should be taken when extrapolating our results to other areas although Beijing is representative of cities with advanced economies and relatively high levels of air pollution.

Although the adverse effect of cerebrovascular disease might be small for each individual, public health implications are very important given the large population with T2D in China exposed to unavoidable air pollution. Further studies with more accurate times of cerebrovascular symptom onset are needed to further validate our findings.

\section{Conclusion}

$\mathrm{NO}_{2}, \mathrm{SO}_{2}, \mathrm{O}_{3}$ and $\mathrm{CO}$ presented nonlinear and lagged effects on cerebrovascular disease among patients with type 2 diabetes. Men were more susceptible to extremely high- $\mathrm{CO}$, and elderly individuals aged over 65 years were susceptible to both extremely low- $\mathrm{O}_{3}$ and high- $\mathrm{CO}$. Our study added to the limited evidence for the risk of comorbidities of T2D and cerebrovascular disease due to gaseous pollutants.

\section{Abbreviations}

T2D: type 2 diabetes; $\mathrm{NO}_{2}$ : nitrogen dioxide; $\mathrm{SO}_{2}$ : sulfur dioxide; $\mathrm{O}_{3}$ : ozone; $\mathrm{CO}$ : carbon monoxide; ICD-10: International Classification of Diseases, $10^{\text {th }}$ Revision; $\mathrm{PM}_{2.5}$ : fine particulate matter with an aerodynamic diameter $<2.5 \mu \mathrm{m} ; \mathrm{PM}_{10}$ : inhalable particulate matter with an aerodynamic diameter $<10 \mu \mathrm{m}$; DLNM: distributed lag nonlinear model; $d f$ : degrees of freedom; 95\% CI: 95\% confidence interval; RR: relative risk.

\section{Declarations}

\section{Acknowledgments}


We acknowledge the Beijing Municipal Health Commission Information Center and the Beijing Air Pollution and Metrological Data Center.

\section{Funding}

This study was supported by National Natural Science Foundation of China (No. 82003559), Nature Science Foundation of Capital Medical University (No. PYZ2018046), Beijing Municipal Training Project of Excellent Talents. The funding was neither used for the study design nor data collection but to cover for the publication fees.

\section{Availability of data and materials}

The data can be accessed from the Beijing Municipal Health Commission Information Center with permission via direct request.

\section{Authors' contributions}

Xiangtong Liu and Zhiwei Li conceived and designed the study. Moning Guo and Feng $\mathrm{Lu}$ advised the retrieval of clinical data. Xiuhua Guo and Lixin Tao supervised the progress of the study. Xiangtong Liu prepared the draft manuscript, and Xiuhua Guo improved the drafted manuscript. Xiangtong Liu, Zhiwei Li, Aklilu Deginet, Mengmeng Liu, Xiaolin $\mathrm{Xu}$, Yanxia Luo, Yaoyu Hu, Yutong Li, Mengqiu Wu and Mengyang Liu advised on the overall data analysis and the development of the manuscript. All authors were involved with the critical revision of the manuscript and approved the final version.

\section{Ethics approval}

In this study, informed consent was not specifically required because we did not use 
460

461

462

463

464

465

466

467

468

469

470

471

472

473

474

475

476

477

478

479

480

481

personal data identifiers. However, the study was approved by the Institutional Review

Board of Capital Medical University with the IRB00009511 identification number.

\section{Consent for publication}

Not applicable.

\section{Competing interests}

The authors declare that they have no competing interests.

\section{References}

Aklilu, D., et al., 2020. Short-term effects of extreme temperatures on cause specific cardiovascular admissions in Beijing, China. Environ Res. 186, 109455.

Balmes, J. R., et al., 2019. Ozone effects on blood biomarkers of systemic inflammation, oxidative stress, endothelial function, and thrombosis: The Multicenter Ozone Study in oldEr Subjects (MOSES). PLoS One. 14, e0222601.

Buddeke, J., et al., 2017. Trends in comorbidity in patients hospitalised for cardiovascular disease. Int J Cardiol. 248, 382-388.

Byrne, C. P., et al., 2020. Short-Term Air Pollution as a Risk for Stroke Admission: A Time-Series Analysis. Cerebrovasc Dis. 1-8.

Chen, C., et al., 2018. Ambient air pollution and daily hospital admissions for mental disorders in Shanghai, China. Sci Total Environ. 613-614, 324-330.

Chen, C., et al., 2019. The effect of air pollution on hospitalization of individuals with respiratory and cardiovascular diseases in Jinan, China. Medicine (Baltimore). 98, e15634.

Chiusolo, M., et al., 2011. Short-Term Effects of Nitrogen Dioxide on Mortality and 

Perspect. 119, 1233-8.

Dionisio, K. L., et al., 2016. A simulation study to quantify the impacts of exposure measurement error on air pollution health risk estimates in copollutant timeseries models. Environ Health. 15, 114.

Feigin, V. L., et al., 2017. Global Burden of Stroke. Circ Res. 120, 439-448.

Feng, W., et al., 2019. Short-term PM10 and emergency department admissions for selective cardiovascular and respiratory diseases in Beijing, China. Sci Total Environ. 657, 213-221.

Gao, J., et al., 2017. Haze, public health and mitigation measures in China: A review of the current evidence for further policy response. Sci Total Environ. 578, 148157.

Gasparrini, A., 2014. Modeling exposure-lag-response associations with distributed lag non-linear models. Stat Med. 33, 881-99.

Guo, Y., et al., 2020. Short-term associations between ambient air pollution and stroke hospitalisations: time-series study in Shenzhen, China. BMJ Open. 10, e032974. Huang, F., et al., 2017. Gaseous Air Pollution and the Risk for Stroke Admissions: A Case-Crossover Study in Beijing, China. Int J Environ Res Public Health. 14.

Li, H., et al., 2018. Effects of ambient carbon monoxide on daily hospitalizations for cardiovascular disease: a time-stratified case-crossover study of 460,938 cases in Beijing, China from 2013 to 2017. Environ Health. 17, 82.

Li, J., et al., 2019. Association between ambient particulate matter air pollution and ST- 
elevation myocardial infarction: A case-crossover study in a Chinese city. Chemosphere. 219, 724-729.

Li, W., et al., 2020. Air quality improvement in response to intensified control strategies in Beijing during 2013-2019. Sci Total Environ. 744, 140776.

Liu, L., et al., 2021. Intraday effects of ambient PM(1) on emergency department visits in Guangzhou, China: A case-crossover study. Sci Total Environ. 750, 142347.

Lokken, R. P., et al., 2009. Air pollution and risk of stroke: underestimation of effect due to misclassification of time of event onset. Epidemiology. 20, 137-42.

Maheswaran, R., et al., 2016. Air Pollution and Subtypes, Severity and Vulnerability to Ischemic Stroke-A Population Based Case-Crossover Study. PLoS One. 11, e0158556.

Miller, M. R., 2020. Oxidative stress and the cardiovascular effects of air pollution. Free Radic Biol Med. 151, 69-87.

Paul, L. A., et al., 2020. The impact of air pollution on the incidence of diabetes and survival among prevalent diabetes cases. Environ Int. 134, 105333.

Shah, A. S., et al., 2015. Short term exposure to air pollution and stroke: systematic review and meta-analysis. Bmj. 350, h1295.

Stafoggia, M., et al., 2017. Statistical Approaches to Address Multi-Pollutant Mixtures and Multiple Exposures: the State of the Science. Curr Environ Health Rep. 4, 481-490.

Sui, X., et al., 2020. The short-term effect of $\mathrm{PM}(2.5) / \mathrm{O}(3)$ on daily mortality from 2013 to 2018 in Hefei, China. Environ Geochem Health. 
Tian, Y., et al., 2018. Association between ambient air pollution and daily hospital admissions for ischemic stroke: A nationwide time-series analysis. PLoS Med. $15, \mathrm{e} 1002668$.

Wang, W., et al., 2017. Prevalence, Incidence, and Mortality of Stroke in China: Results from a Nationwide Population-Based Survey of 480687 Adults. Circulation. $135,759-771$.

Wang, X. D., et al., 2016. Short-term effects of air pollution on acute myocardial infarctions in Shanghai, China, 2013-2014. J Geriatr Cardiol. 13, 132-7.

Wang, Z., et al., 2020. Association between short-term exposure to air pollution and ischemic stroke onset: a time-stratified case-crossover analysis using a distributed lag nonlinear model in Shenzhen, China. Environ Health. 19, 1.

Yang, Y., et al., 2018. Ambient fine particulate pollution associated with diabetes mellitus among the elderly aged 50 years and older in China. Environ Pollut. $243,815-823$.

Zhou, M., et al., 2016. Cause-specific mortality for 240 causes in China during 19902013: a systematic subnational analysis for the Global Burden of Disease Study 2013. Lancet. 387, 251-72. 


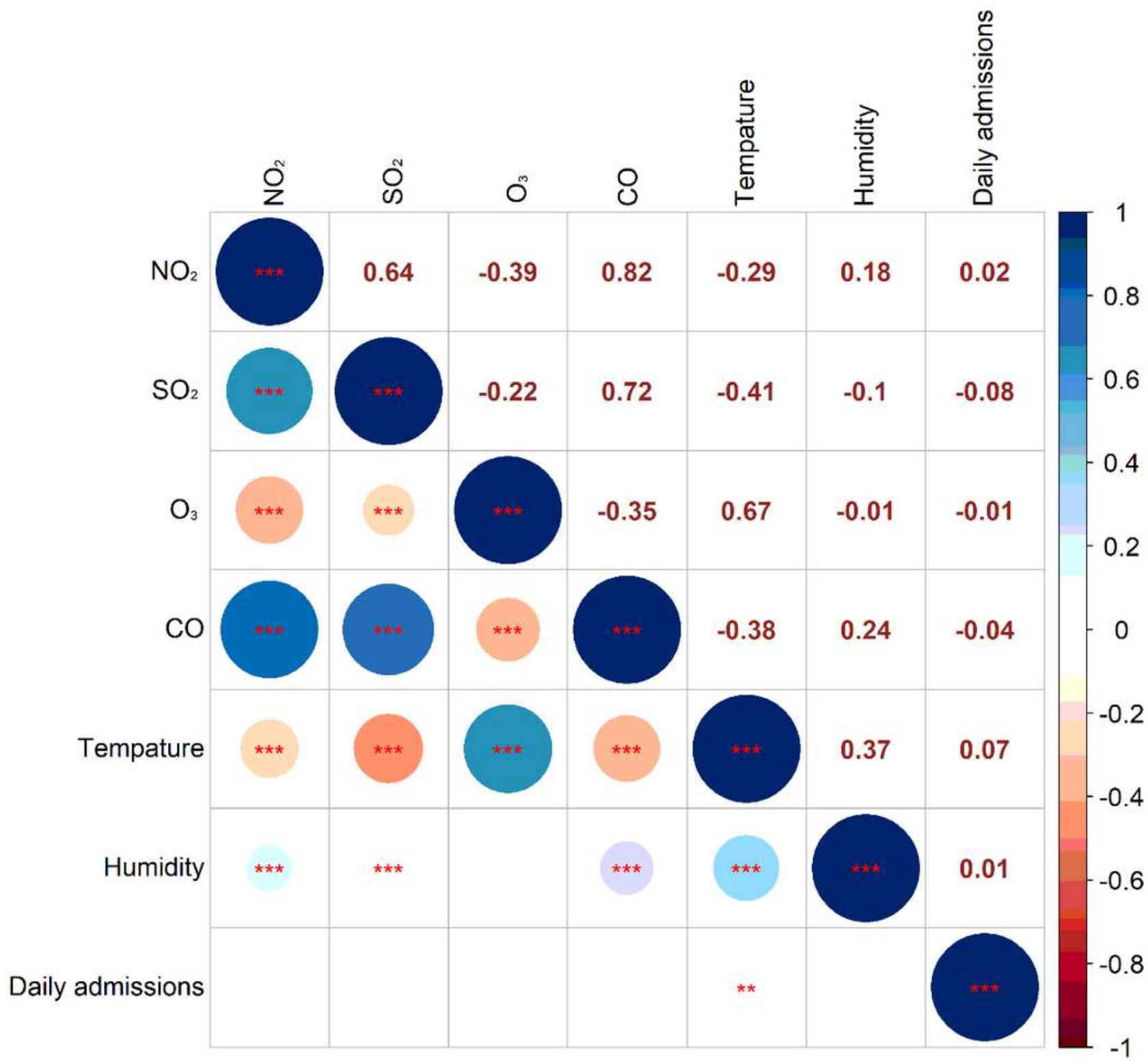

\section{Figure 1}

Pearson correlation coefficients between gaseous air pollutants concentrations and weather conditions and daily admissions for cerebrovascular disease in Beijing, 2014-2018 $(n=1799)$. NO2: nitrogen dioxide; SO2: sulfur dioxide; O3: ozone; CO: carbon monoxide. ${ }^{*} 0.01<\mathrm{P} \leq 0.05 ; * \star 0.001<\mathrm{P} \leq 0.01 ; * \star \star \mathrm{P} \leq 0.001$. 
(a) $\mathrm{SO}_{2}$

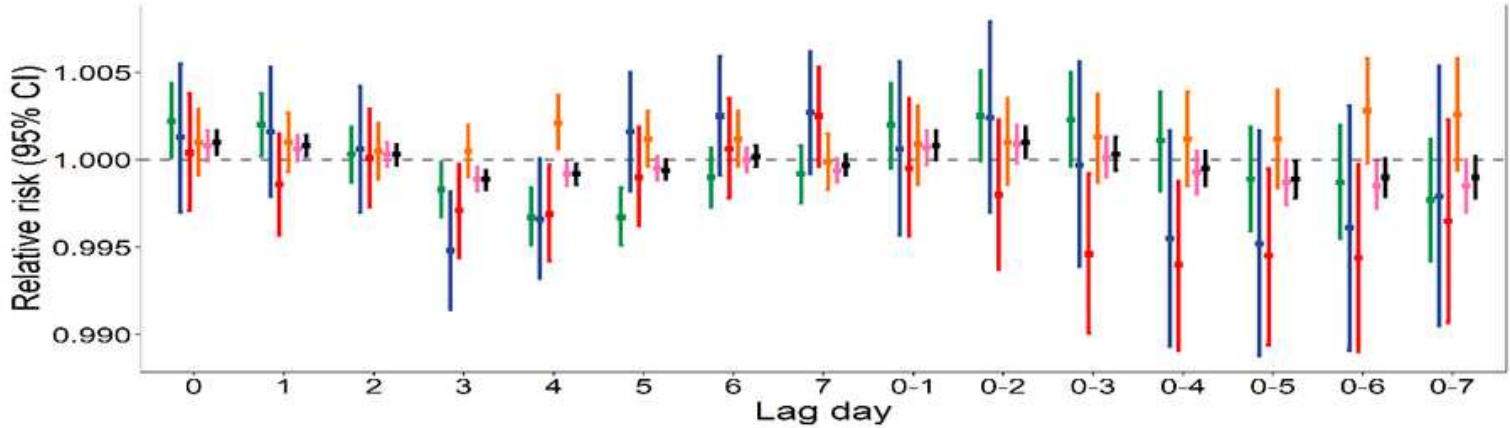

(b) $\mathrm{NO}_{2}$

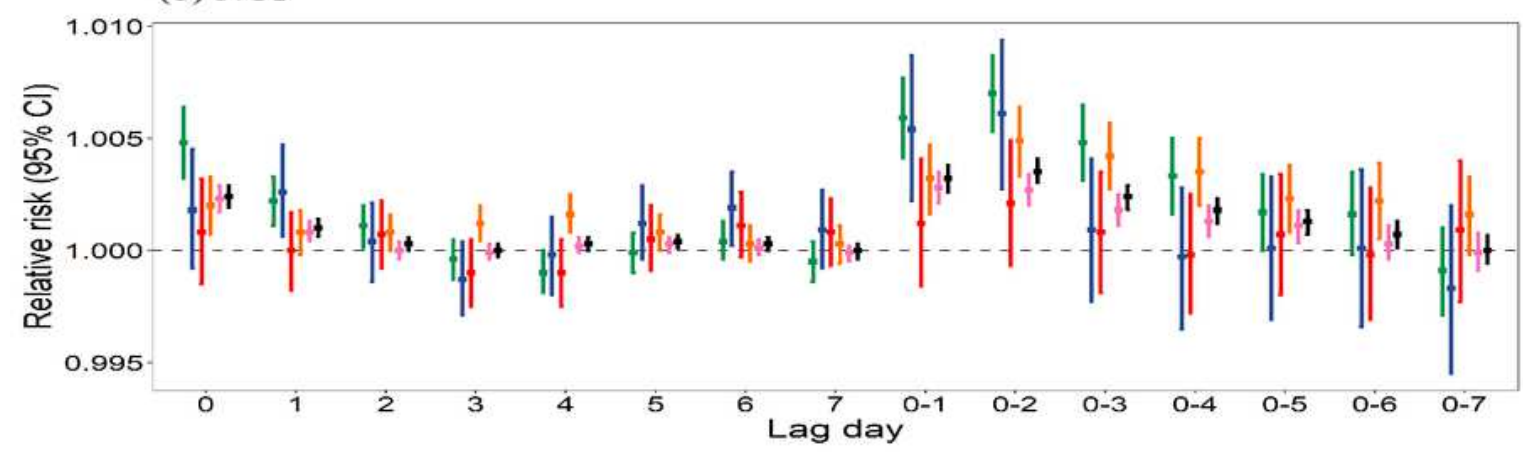

(c) $\mathrm{O}_{3}$
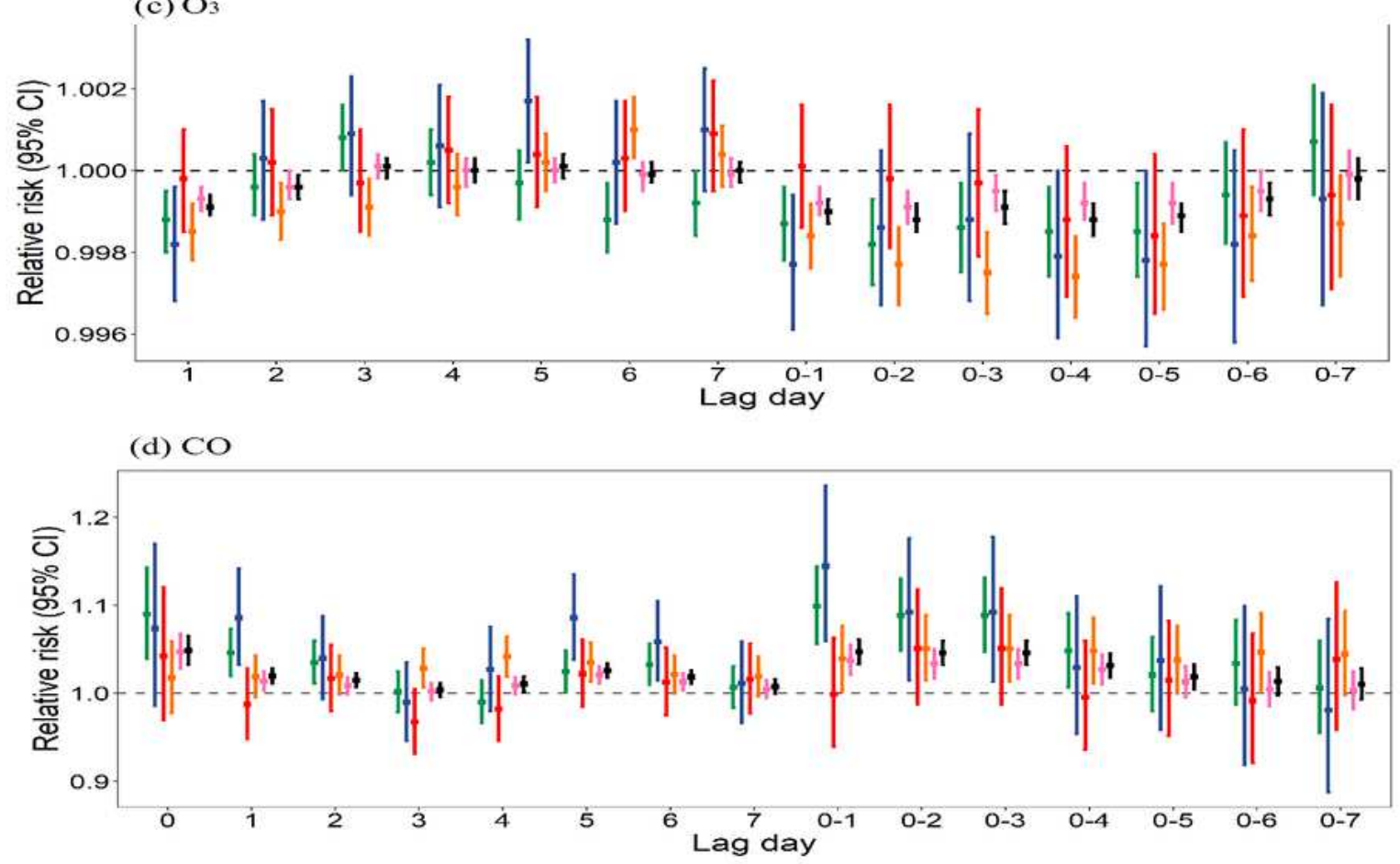

Figure 2

Relative risk with $95 \% \mathrm{Cl}$ (confidence interval) in cerebrovascular morbidity associated with increase in concentrations of gaseous pollutants for different lag structures in multi-pollutant model: (a) SO2: sulfur dioxide; (b) NO2: nitrogen dioxide; (c) 03: ozone; (d) CO: carbon monoxide. 
(a) Overall effect of $\mathrm{NO}_{2}$ on CBVD

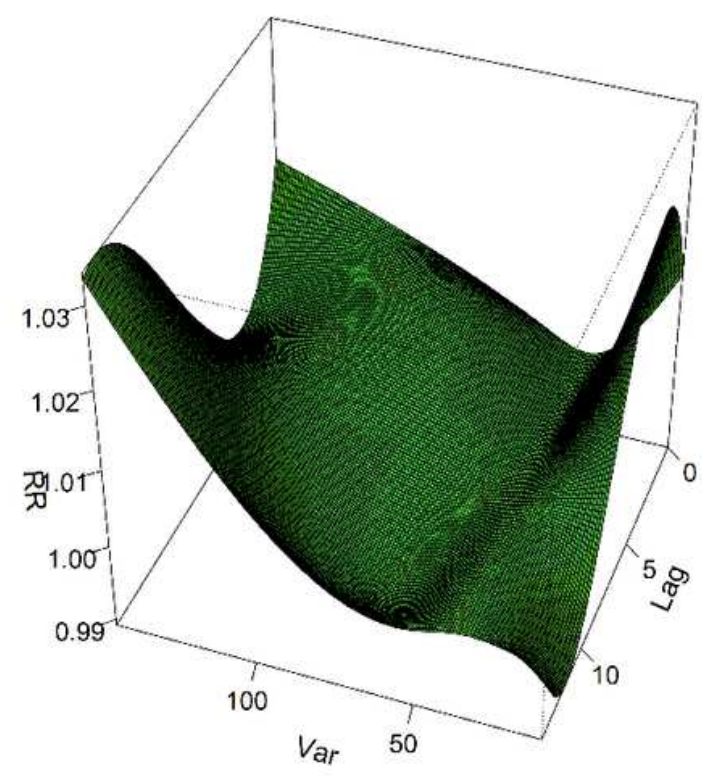

(c) Overall effect of $\mathrm{O}_{3}$ on CBVD

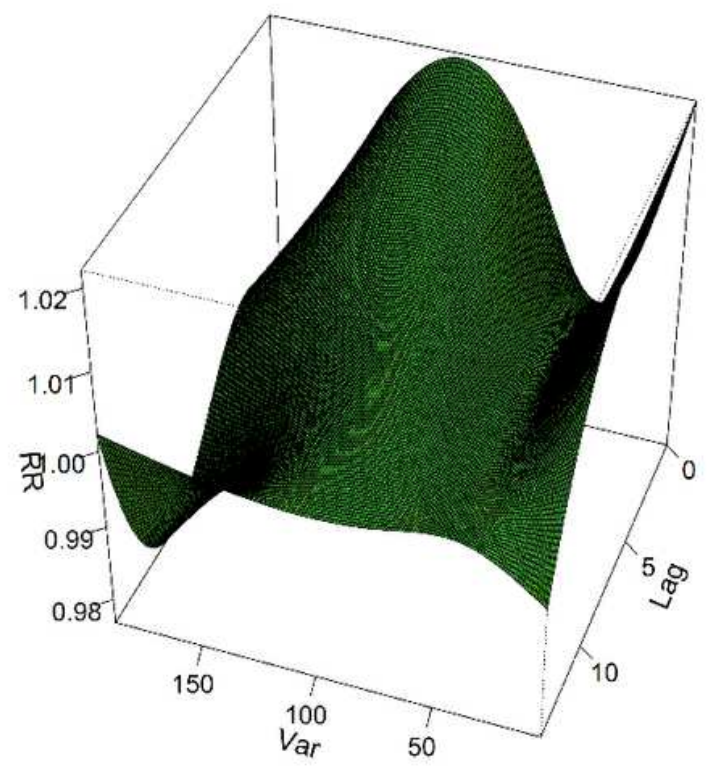

(b) Overall effect of $\mathrm{sO}_{2}$ on CBVD

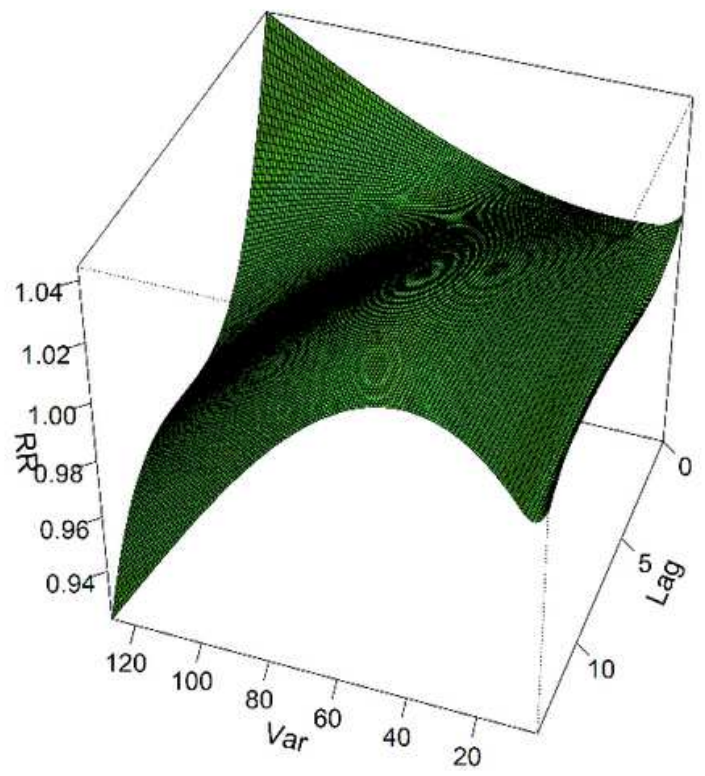

(d) Overall effect of $\mathrm{CO}$ on CBVD

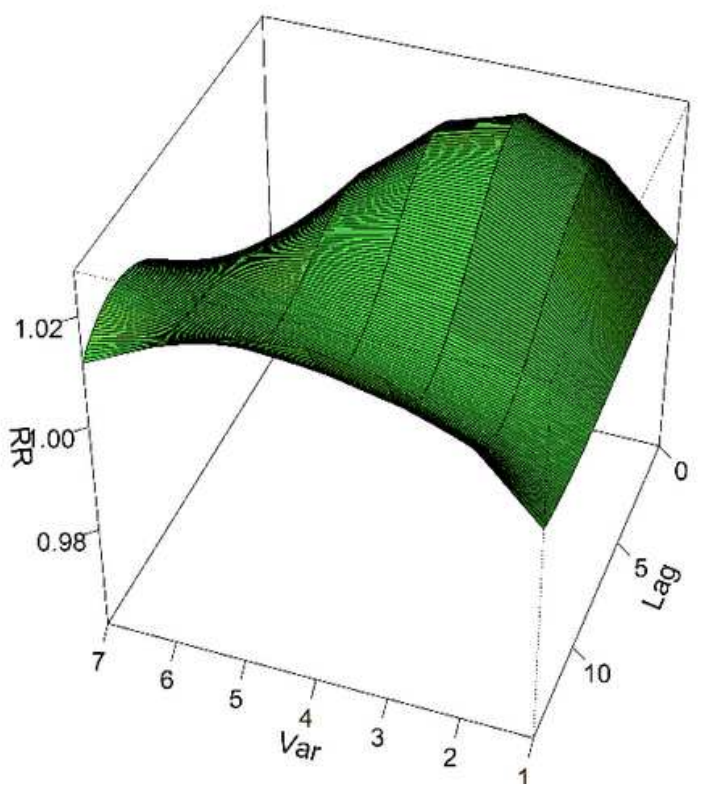

\section{Figure 3}

3D graphs of NO2, SO2, 03 and $\mathrm{CO}$ effect on overall cerebrovascular cases: (a) overall effect of NO2; (b) overall effect of SO2; (c) overall effect of O3; (d) overall effect of CO. NO2: nitrogen dioxide; SO2: sulfur dioxide; 03: ozone; CO: carbon monoxide. RR (95\% Cl): relative risk and 95\% confidence interval. 
(a) Cumulative effect of $\mathrm{NO}_{2}$

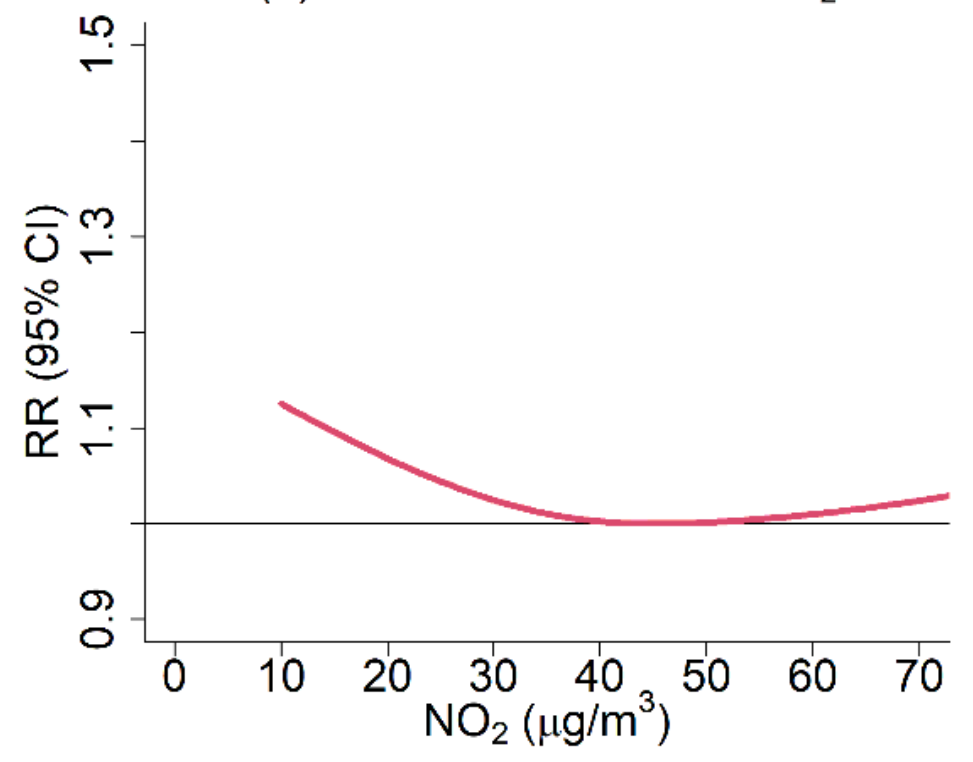

(c) Cumulative effect of $\mathrm{O}_{3}$

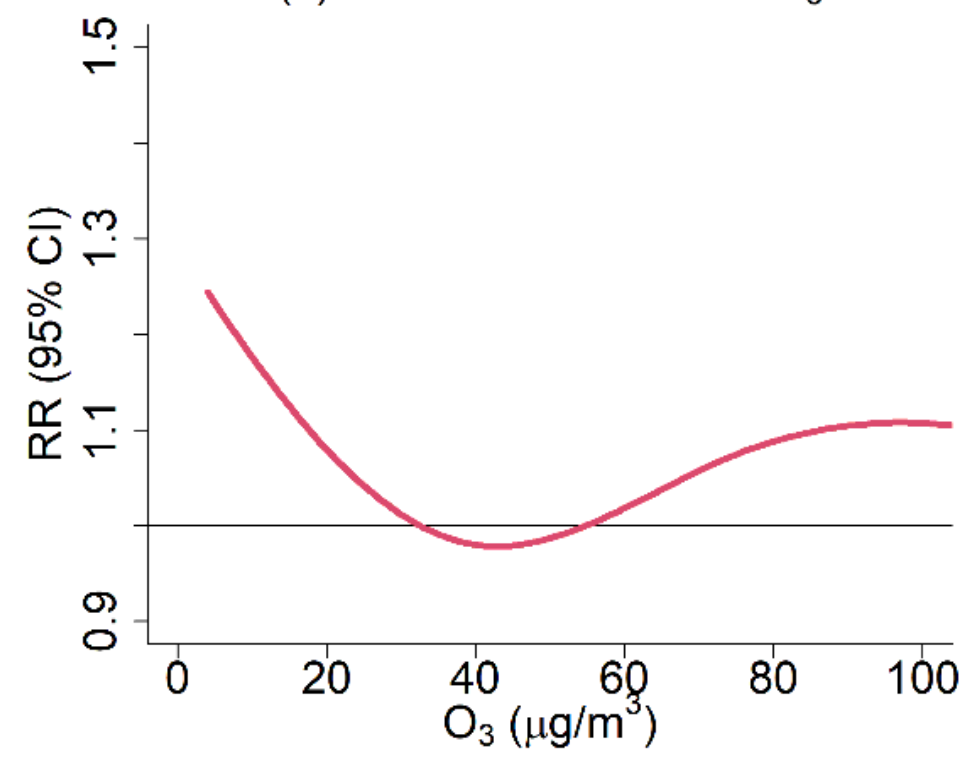

(b) Cumulative effect of $\mathrm{SO}_{2}$

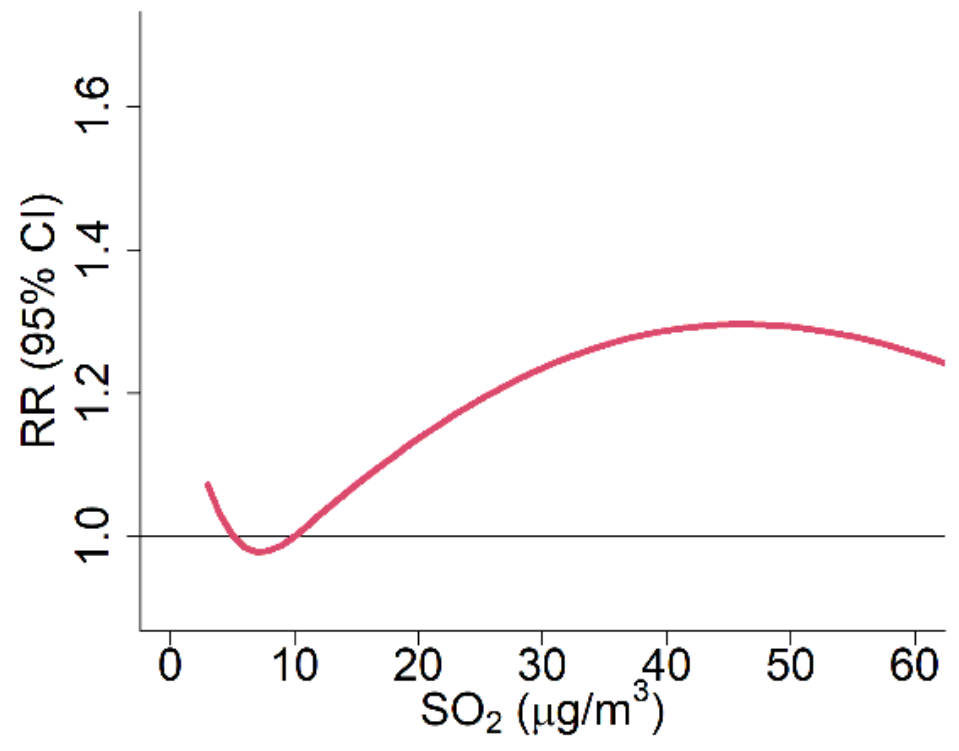

(d) Cumulative effect of $\mathrm{CO}$

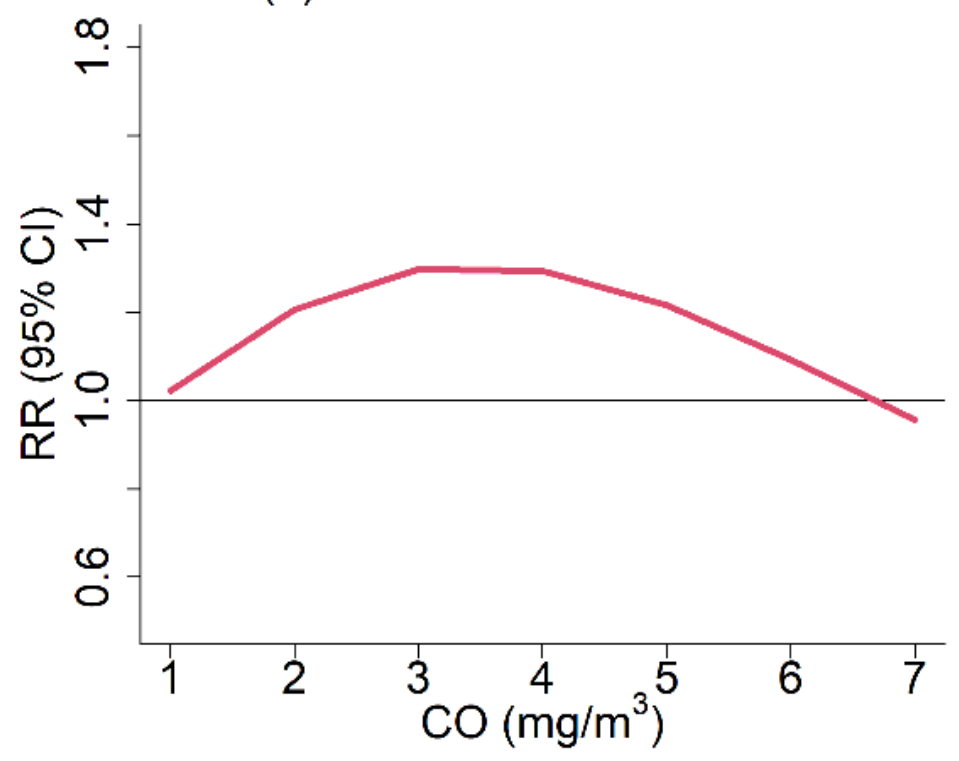

\section{Figure 4}

Cumulative exposure-response curves on overall cerebrovascular cases for gaseous pollutants (NO2, SO2, 03 and CO) at lag0-7 using multi-pollutant models in Beijing, 2014-2018: (a) cumulative effect of $\mathrm{NO} 2$; (b) cumulative effect of SO2; (c) cumulative effect of 03; (d) cumulative effect of $\mathrm{CO}$. NO2: nitrogen dioxide; SO2: sulfur dioxide; O3: ozone; CO: carbon monoxide. RR (95\% Cl): relative risk and 95\% confidence interval. 


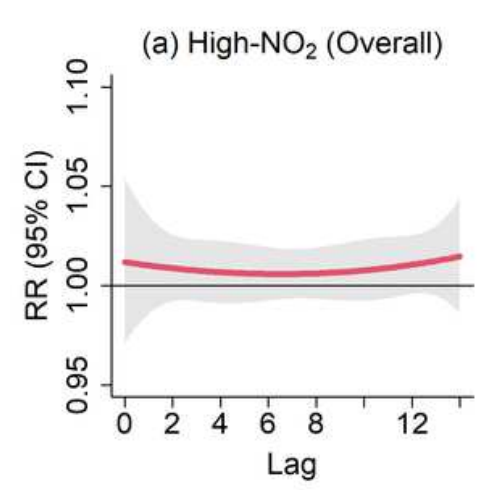

(e) Low- $\mathrm{NO}_{2}$ (Overall)

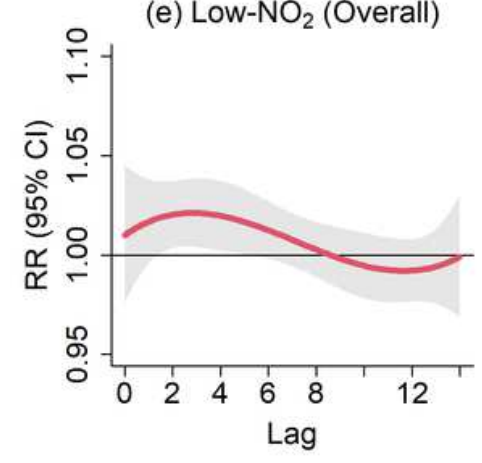

(b) $\mathrm{High}-\mathrm{SO}_{2}$ (Overall)

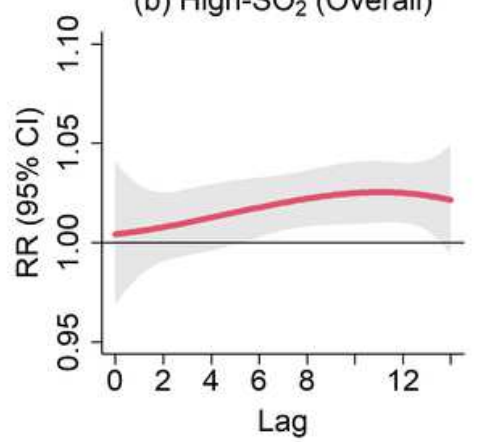

(f) Low- $\mathrm{SO}_{2}$ (Overall)

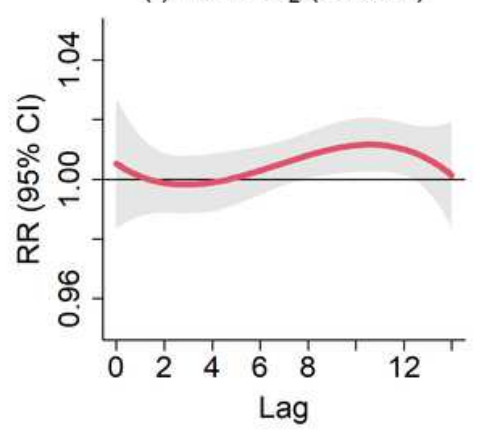

(c) High- $\mathrm{O}_{3}$ (Overall)

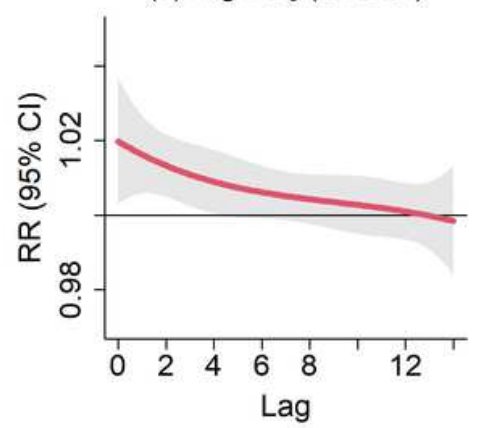

(g) Low-O $\mathrm{O}_{3}$ (Overall)

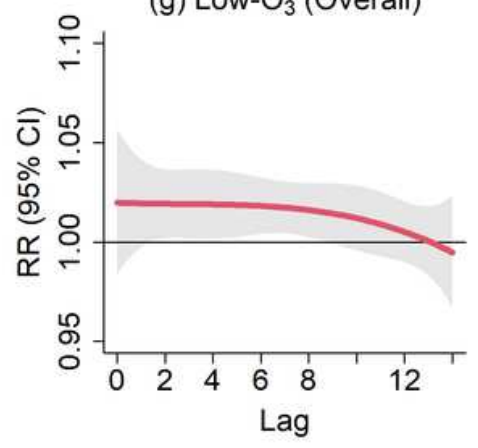

(d) High-CO (Overall)

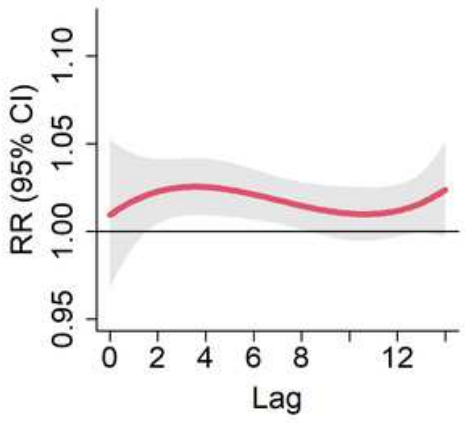

(h) Low-CO (Overall)

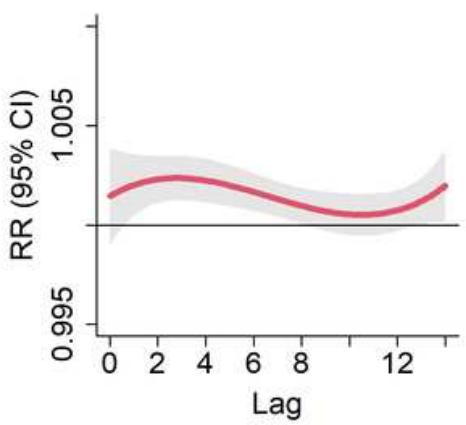

Figure 5

Single day lag-response curves on overall cerebrovascular cases for extreme gaseous pollutants concentrations using multi-pollutant model in Beijing, 2014-2018: (a) overall effect of extreme high-NO2; (b) overall effect of extreme high-SO2; (c) overall effect of extreme high-03; (d) overall effect of extreme high-CO; (e) overall effect of extreme low-NO2; (f) overall effect of extreme low-SO2; (g) overall effect of extreme low-03; (h) overall effect of extreme low-CO. The high effect was estimated by the RR of cerebrovascular disease by comparing the 99th percentile to the median of daily concentration, whereas the low effect was estimated by comparing the $1 \mathrm{st}$ percentile to the median of daily concentration. NO2: nitrogen dioxide; SO2: sulfur dioxide; O3: ozone; CO: carbon monoxide. RR (95\% Cl): relative risk and 95\% confidence interval.

\section{Supplementary Files}

This is a list of supplementary files associated with this preprint. Click to download.

- 3supplementalfile21.4.1.docx 